\title{
Global time evolution of concentrated vortex rings
}

\author{
Paolo Buttà, Guido Cavallaro and Carlo Marchioro
}

\begin{abstract}
We study the time evolution of an incompressible fluid with axial symmetry without swirl, assuming initial data such that the initial vorticity is very concentrated inside $N$ small disjoint rings of thickness $\varepsilon$ and vorticity mass of the order of $|\log \varepsilon|^{-1}$. When $\varepsilon \rightarrow 0$, we show that the motion of each vortex ring converges to a simple translation with constant speed (depending on the single ring) along the symmetry axis. We obtain a sharp localization of the vorticity support at time $t$ in the radial direction, whereas we state only a concentration property in the axial direction. This is obtained for arbitrary (but fixed) intervals of time. This study is the completion of a previous paper [5], where a sharp localization of the vorticity support was obtained both along the radial and axial directions, but the convergence for $\varepsilon \rightarrow 0$ worked only for short times.
\end{abstract}

Mathematics Subject Classification. 76B47, 37N10.

Keywords. Incompressible Euler flow, Vortex rings.

\section{Introduction and main result}

We study the time evolution of an incompressible non-viscous fluid in the whole space $\mathbb{R}^{3}$, with an axial symmetry without swirl when the vorticity is sharply concentrated on $N$ annuli of radii $r_{i} \approx r_{0}$ and thickness $\varepsilon$. In particular, we consider the limit $\varepsilon \rightarrow 0$. In a previous paper of some years ago, a similar problem [3] was investigated for a vortex alone, showing that it translates with a constant speed. Recently, in [5], the analysis has been extended to the case of $N$ vortices, also getting a stronger localization property, but restricted to the case of short but positive time. In the present paper, we study the problem for any time.

The motion of an incompressible inviscid fluid is governed by the Euler equations that for a fluid of unitary density in three dimensions with velocity $\boldsymbol{u}=\boldsymbol{u}(\boldsymbol{\xi}, t)$ decaying at infinity read

$$
\begin{aligned}
& \partial_{t} \boldsymbol{\omega}+(\boldsymbol{u} \cdot \nabla) \boldsymbol{\omega}=(\boldsymbol{\omega} \cdot \nabla) \boldsymbol{u} \\
& \boldsymbol{u}(\boldsymbol{\xi}, t)=-\frac{1}{4 \pi} \int_{\mathbb{R}^{3}} \mathrm{~d} \boldsymbol{\eta} \frac{(\boldsymbol{\xi}-\boldsymbol{\eta}) \wedge \boldsymbol{\omega}(\boldsymbol{\eta}, t)}{|\boldsymbol{\xi}-\boldsymbol{\eta}|^{3}},
\end{aligned}
$$

where $\boldsymbol{\omega}=\boldsymbol{\omega}(\boldsymbol{\xi}, t)=\nabla \wedge \boldsymbol{u}(\boldsymbol{\xi}, t)$ is the vorticity, $\boldsymbol{\xi}=\left(\xi_{1}, \xi_{2}, \xi_{3}\right)$ denotes a point in $\mathbb{R}^{3}$, and $t \in \mathbb{R}_{+}$is the time. The equations are completed by the initial conditions. It is worthwhile to emphasize that the incompressibility condition $\nabla \cdot \boldsymbol{u}=0$ is clearly verified in view of Eq. (1.2).

Denoting by $(z, r, \theta)$ the cylindrical coordinates, we recall that the vector field $\boldsymbol{F}$ of cylindrical components $\left(F_{z}, F_{r}, F_{\theta}\right)$ is called axisymmetric without swirl if $F_{\theta}=0$ and $F_{z}$ and $F_{r}$ are independent of $\theta$.

The axisymmetry is preserved by the evolution Eqs. (1.1), (1.2). Moreover, when restricted to axisymmetric velocity fields $\boldsymbol{u}(\boldsymbol{\xi}, t)=\left(u_{z}(z, r, t), u_{r}(z, r, t), 0\right)$, the vorticity is

$$
\boldsymbol{\omega}=\left(0,0, \omega_{\theta}\right)=\left(0,0, \partial_{z} u_{r}-\partial_{r} u_{z}\right)
$$


and, denoting henceforth $\omega_{\theta}$ by $\omega$, Eq. (1.1) reduces to

$$
\partial_{t} \omega+\left(u_{z} \partial_{z}+u_{r} \partial_{r}\right) \omega-\frac{u_{r} \omega}{r}=0 .
$$

Finally, by Eq. (1.2), $u_{z}=u_{z}(z, r, t)$ and $u_{r}=u_{r}(z, r, t)$ are given by

$$
\begin{aligned}
& u_{z}=-\frac{1}{2 \pi} \int \mathrm{d} z^{\prime} \int_{0}^{\infty} r^{\prime} \mathrm{d} r^{\prime} \int_{0}^{\pi} \mathrm{d} \theta \frac{\omega\left(z^{\prime}, r^{\prime}, t\right)\left(r \cos \theta-r^{\prime}\right)}{\left[\left(z-z^{\prime}\right)^{2}+\left(r-r^{\prime}\right)^{2}+2 r r^{\prime}(1-\cos \theta)\right]^{3 / 2}}, \\
& u_{r}=\frac{1}{2 \pi} \int \mathrm{d} z^{\prime} \int_{0}^{\infty} r^{\prime} \mathrm{d} r^{\prime} \int_{0}^{\pi} \mathrm{d} \theta \frac{\omega\left(z^{\prime}, r^{\prime}, t\right)\left(z-z^{\prime}\right) \cos \theta}{\left[\left(z-z^{\prime}\right)^{2}+\left(r-r^{\prime}\right)^{2}+2 r r^{\prime}(1-\cos \theta)\right]^{3 / 2}} .
\end{aligned}
$$

In conclusion, the axisymmetric solutions to the Euler equations are the solutions to Eqs. (1.4), (1.5), and (1.6).

We notice that Eq. (1.4) means that the quantity $\omega / r$ remains constant along the flow generated by the velocity field, i.e.,

$$
\frac{\omega(z(t), r(t), t)}{r(t)}=\frac{\omega(z(0), r(0), 0)}{r(0)}
$$

with $(z(t), r(t))$ solution to

$$
\dot{z}(t)=u_{z}(z(t), r(t), t), \quad \dot{r}(t)=u_{r}(z(t), r(t), t) .
$$

We notice that in the case of non-smooth initial data, Eqs. (1.5), (1.6), (1.7), and (1.8) can be assumed as a weak formulation of the Euler equations in the framework of axisymmetric solutions. An equivalent weak formulation is obtained from Eq. (1.4) by a formal integration by parts,

$$
\frac{\mathrm{d}}{\mathrm{d} t} \omega_{t}[f]=\omega_{t}\left[u_{z} \partial_{z} f+u_{r} \partial_{r} f+\partial_{t} f\right]
$$

where $f=f(z, r, t)$ is any bounded smooth test function and

$$
\omega_{t}[f]:=\int \mathrm{d} z \int_{0}^{\infty} \mathrm{d} r \omega(z, r, t) f(z, r, t) .
$$

It is known that the global (in time) existence and uniqueness of a weak solution to the associate Cauchy problem holds when initial vorticity is a bounded function with compact support contained in the open half-plane $\Pi:=\{(z, r): r>0\}$, see, for instance, [21, Page 91] or [7, Appendix]. In particular, it can be shown that the support of the vorticity remains in the open half-plane $\Pi$ at any time (note that a point in the half-plane $\Pi$ corresponds to a circumference in the three dimensional space $\mathbb{R}^{3}$ ).

The special class of axisymmetric solutions without swirl is known in the literature as smoke rings (or vortex rings), because of the property to preserve their shape in time, which translates with a constant speed along the $z$-axis. The knowledge of such solutions is very old, but a first rigorous proof of existence and properties of these solutions (in the stationary case) goes back to [1,10], by means of variational methods. Other information and references on axially symmetric solution without swirl can be found in $[22]$.

We consider in the present paper the special class of initial data for which the vorticity is initially very concentrated. We mean that, given a small parameter $\varepsilon \in(0,1)$, we take initial data for which the vorticity has compact support contained in $N$ disks, that is

$$
\omega_{\varepsilon}(z, r, 0)=\sum_{i=1}^{N} \omega_{i, \varepsilon}(z, r, 0),
$$


where $\omega_{i, \varepsilon}(z, r, 0), i=1, \ldots, N$, are functions with definite sign whose support is contained in $\Sigma\left(\zeta^{i} \mid \varepsilon\right)$, which is the open disk of center $\zeta^{i}$ and radius $\varepsilon$,

$$
\Lambda_{i, \varepsilon}(0):=\operatorname{supp} \omega_{i, \varepsilon}(\cdot, 0) \subset \Sigma\left(\zeta^{i} \mid \varepsilon\right),
$$

with

$$
\overline{\Sigma\left(\zeta^{i} \mid \varepsilon\right)} \subset \Pi \quad \forall i, \quad \Sigma\left(\zeta^{i} \mid \varepsilon\right) \cap \Sigma\left(\zeta^{j} \mid \varepsilon\right)=\emptyset \quad \forall i \neq j,
$$

for fixed $\zeta^{i}=\left(z_{i}, r_{i}\right) \in \Pi$. We assume also that

$$
\min _{i} r_{i}>2 D \quad \forall i, \quad\left|r_{i}-r_{j}\right| \geq 2 D \quad \forall i \neq j
$$

where $D$ is a positive fixed constant. This means that the annuli have different radii, which is an essential hypothesis as explained in Remark 1.1 below.

In view of (1.7), the decomposition Eq. (1.10) extends to positive time setting

$$
\omega_{\varepsilon}(z, r, t)=\sum_{i=1}^{N} \omega_{i, \varepsilon}(z, r, t)
$$

with $\omega_{i, \varepsilon}(x, t)$ being the time evolution of the $i$ th vortex ring,

$$
\omega_{i, \varepsilon}(z(t), r(t), t):=\frac{r(t)}{r(0)} \omega_{\varepsilon, i}(z(0), r(0), 0) .
$$

We focus on the case of a fluid with a large vorticity concentration. Therefore, in order to have nontrivial (i.e., neither vanishing nor diverging) limiting velocities of the vortex rings, the initial data have to be chosen appropriately. The correct choice can be inferred by considering the simplest case of a vortex ring alone, of intensity $N_{\varepsilon}=: \int \mathrm{d} z \int_{0}^{\infty} \mathrm{d} r \omega_{\varepsilon}(z, r, 0)$ and supported in a small region of diameter $\varepsilon$. It is well known that it moves along the $z$-direction with an approximately constant speed proportional to $N_{\varepsilon}|\log \varepsilon|$, see [9]. With this in mind, we assume that there are $N$ real parameters $a_{1}, \ldots, a_{N}$, called vortex intensities, such that

$$
|\log \varepsilon| \int \mathrm{d} z \int_{0}^{\infty} \mathrm{d} r \omega_{i, \varepsilon}(z, r, 0)=a_{i} \quad \forall i=1, \ldots, N .
$$

Finally, to avoid too large vorticity concentrations, we further assume there is a constant $M>0$ such that

$$
\left|\omega_{i, \varepsilon}(z, r, 0)\right| \leq \frac{M}{\varepsilon^{2}|\log \varepsilon|} \quad \forall(z, r) \in \Pi \quad \forall i=1, \ldots, N .
$$

Now, we can state the main result of the paper.

Theorem 1.1. Assume the initial data $\omega_{\varepsilon}(x, 0)$ verify Eqs. (1.10), (1.11), (1.12), (1.15), and (1.16), and define

$$
\zeta^{i}(t):=\zeta^{i}+\frac{a_{i}}{4 \pi r_{i}}\left(\begin{array}{l}
1 \\
0
\end{array}\right) t, \quad i=1, \ldots, N .
$$

Then, for any $T>0$ the following holds true. For any $\varepsilon$ small enough there are $\zeta^{i, \varepsilon}(t) \in \Pi, t \in[0, T]$, $i=1, \ldots, N$, and $R_{\varepsilon}>0$ such that

$$
\lim _{\varepsilon \rightarrow 0}|\log \varepsilon| \int_{\Sigma\left(\zeta^{i, \varepsilon}(t) \mid R_{\varepsilon}\right)} \mathrm{d} z \mathrm{~d} r \omega_{i, \varepsilon}(z, r, t)=a_{i} \quad \forall i=1, \ldots, N, \quad \forall t \in[0, T],
$$

with

$$
\lim _{\varepsilon \rightarrow 0} R_{\varepsilon}=0, \quad \lim _{\varepsilon \rightarrow 0} \zeta^{i, \varepsilon}(t)=\zeta^{i}(t) \quad \forall t \in[0, T] .
$$


Remark 1.1. As already mentioned, the assumption Eq. (1.12), which guarantees $\left|\zeta^{i}(t)-\zeta^{j}(t)\right| \geq 2 D$ for any $i \neq j$ and $t \geq 0$, is essential. Indeed, letting $\Lambda_{i, \varepsilon}(t):=\operatorname{supp} \omega_{i, \varepsilon}(\cdot, t), i=1, \ldots, N$, a key point in the proof is to show that $\Lambda_{i, \varepsilon}(t) \cap \Lambda_{i, \varepsilon}(t)=\emptyset$ for any $i \neq j$ and $t \geq 0$. This holds because, as long as the supports remain separated, their size along the $r$-direction can be proved to remain infinitesimal as $\varepsilon \rightarrow 0$. On the other hand, we are not able to get an analogous strong localization property for the size of $\Lambda_{i, \varepsilon}(t)$ in the $z$-direction. Therefore, without the assumption Eq. (1.12) we cannot prevent an overlap of the supports of different rings in a finite time.

We emphasize that our analysis concerns an asymptotic regime $\varepsilon \rightarrow 0$ in which the interaction between different rings actually vanishes, so that the limiting motion of each ring is not influenced by the other ones. Features of the dynamics for finite $\varepsilon$, as the leapfrogging phenomenon, although very interesting, are beyond the scope of the present paper.

Remark 1.2. Sometimes, the Euler equations are considered with initial vorticity highly concentrated around a generic curve, say $\Gamma=\left\{\gamma_{\sigma}\right\}_{\sigma \in[0,1]} \subset \mathbb{R}^{3}$, see, e.g., [15]. Of course, additional assumptions are needed to analyze the time evolution. Here, the main feature is the so-called LIA approximation (local induction approximation), in which the vorticity remains concentrated around a vortex filament $\Gamma(t)=\left\{\gamma_{\sigma}(t)\right\}_{\sigma \in[0,1]}$, whose time velocity $\dot{\gamma}_{\sigma}(t)$ depends on the curvature and is directed along the bi-normal vector. Of course, the case of circular rings with full axial symmetry considered here can be thought as a very special example of these phenomena.

Remark 1.3. The effect of a viscosity perturbation in the derivation of the vortex model has been discussed in the literature $[2,7,12-14,16,17,20]$, but this topic is out of the purposes of the present analysis.

Remark 1.4. In this paper, we show that for certain classes of concentrated initial data the time evolution is closely related to the dynamics of a particular system of particles. This is mainly related to the choice of $r_{0}$, which is the typical distance of the rings from the symmetry axis. It is worthwhile to mention that different choices of $r_{0}$ have been already considered in the literature. More precisely, if $r_{0}$ is sufficiently large, i.e., $r_{0} \approx \varepsilon^{-\alpha}, \alpha>0$, the centers of vorticity converge for $\varepsilon \rightarrow 0$ to the solution of the point vortex system, see [18]. This result has been extended in [6] up to the case $r_{0} \approx|\log \varepsilon|^{\alpha}$ with $\alpha>2$. The more interesting regime is $r_{0} \approx|\log \varepsilon|$, where the limiting motion of the vorticity centers is conjectured to be governed by a dynamical system which is a combination of point vortex dynamics and simple translation, see [19] where only convergence at time zero is proved. Actually, the relation between the solutions of the Euler equations and time evolution of special particle systems is more general and it is at the basis of an approximation method, called "vortex method," widely used in literature, see, e.g., [8] or the textbook $[21]$.

The strategy in the proof of Theorem 1.1 is the same of the previous works on the topics. (We quote here only the more recent ones [4,5], and address the reader to the references therein). We first show the corresponding result for a "reduced system," where a vortex ring alone moves under the action of a suitable external time-dependent vector field. The result for the original model is then achieved by treating the motion of each vortex ring as that of a reduced system, in which the external field describes the force due to its interaction with the other rings.

The key tool in the planar case [4] is a sharp a priori estimate on the moment of inertia, which is not available in the axial symmetric case because the velocity field is not a Lipschitz function. To overcome this problem, in [5] the "quasi" energy conservation is used to control the growth in time of the moment of inertia, which allows us to build up an iterative scheme to deduce the sharp localization property, but the price to pay is that this scheme converges only for short times.

Theorem 1.1 extends the result of [5] globally in time, and the strategy behind this improvement relies in the following observation. A suitable decomposition of the velocity field shows that its non-Lipschitz part is directed along the $z$-axis, which suggests that the vorticity should stay more localized along the radial direction (as already discussed in Remark 1.1). Indeed, this is true and allows us to deduce an 
estimate on a different quantity, the "axial moment of inertia." This new estimate, together with the lack of the non-Lipschitz term affecting the radial motion, makes possible to build up an iterative scheme as in [5], but here convergent at any positive time, thus deducing a sharp localization property globally in time.

The plan of the paper is the following. In the next section, we introduce the reduced system and prove Theorem 1.1 as a corollary of the analogous result for this system, which is proved in Sects. 3 and 4 . In "Appendix A" we extend to the reduced system a concentration property of the vorticity distribution, proved in [3] for the case of a vortex alone. This property is necessary to characterize the axial motion and its proof relies on an accurate control on the time variation of the energy. Finally, in Appendix B, we prove Lemma 2.3, which shows how the motion of each vortex ring coincides with that of a suitable reduced system.

\section{Reduction to a single vortex problem}

We rename the variables by letting

$$
x=\left(x_{1}, x_{2}\right):=(z, r)
$$

and extend the vorticity to a function on the whole plane by setting $\omega_{\varepsilon}(x, t)=0$ for $x_{2} \leq 0$, so that $x=\left(x_{1}, x_{2}\right) \in \mathbb{R}^{2}$ henceforth. In this way, the equations of motion Eqs. (1.5), (1.6), (1.7), and (1.8) take the following form,

$$
\begin{aligned}
& u(x, t)=\int \mathrm{d} y H(x, y) \omega_{\varepsilon}(y, t), \\
& \omega_{\varepsilon}(x(t), t)=\frac{x_{2}(t)}{x_{2}(0)} \omega_{\varepsilon}(x(0), 0), \\
& \dot{x}(t)=u(x(t), t),
\end{aligned}
$$

where $u(x, t)=\left(u_{1}(x, t), u_{2}(x, t)\right)$ and the kernel $H(x, y)=\left(H_{1}(x, y), H_{2}(x, y)\right)$ is given by

$$
\begin{aligned}
& H_{1}(x, y)=\frac{1}{2 \pi} \int_{0}^{\pi} \mathrm{d} \theta \frac{y_{2}\left(y_{2}-x_{2} \cos \theta\right)}{\left[|x-y|^{2}+2 x_{2} y_{2}(1-\cos \theta)\right]^{3 / 2}}, \\
& H_{2}(x, y)=\frac{1}{2 \pi} \int_{0}^{\pi} \mathrm{d} \theta \frac{y_{2}\left(x_{1}-y_{1}\right) \cos \theta}{\left[|x-y|^{2}+2 x_{2} y_{2}(1-\cos \theta)\right]^{3 / 2}} .
\end{aligned}
$$

The "reduced system" describes the motion of a single vortex ring in a suitable external time-dependent vector field, which simulates the interaction with the other vortices. This system is defined by Eqs. (2.2), (2.3), and, in place of Eq. (2.4),

$$
\dot{x}(t)=u(x(t), t)+F^{\varepsilon}(x(t), t) .
$$

The initial datum $\omega_{\varepsilon}(x, 0)$ and the time-dependent vector field $F^{\varepsilon}$ are assumed to satisfy the following conditions.

Assumption 2.1. The function $\omega_{\varepsilon}(x, 0)$ is nonnegative (resp. non-positive) and there is $M>0$ and $a>0$ (resp. $a<0$ ) such that

$$
0 \leq\left|\omega_{\varepsilon}(x, 0)\right| \leq \frac{M}{\varepsilon^{2}|\log \varepsilon|} \quad \forall x \in \mathbb{R}^{2}, \quad|\log \varepsilon| \int \mathrm{d} y \omega_{\varepsilon}(y, 0)=a .
$$

Moreover, there exists $\zeta^{0}=\left(z_{0}, r_{0}\right)$, with $r_{0}>0$, such that

$$
\Lambda_{\varepsilon}(0):=\operatorname{supp} \omega_{\varepsilon}(\cdot, 0) \subset \Sigma\left(\zeta^{0} \mid \varepsilon\right) .
$$


Finally, $F^{\varepsilon}=\left(F_{1}^{\varepsilon}, F_{2}^{\varepsilon}\right)$ is a continuous and globally Lipschitz vector field, and it enjoys the following properties.

(a) $\partial_{x_{1}}\left(x_{2} F_{1}^{\varepsilon}\right)+\partial_{x_{2}}\left(x_{2} F_{2}^{\varepsilon}\right)=0$. Otherwise stated, if we denote by $\boldsymbol{F}^{\varepsilon}$ the three-dimensional vector field whose components in cylindrical coordinates are $\left(F_{z}^{\varepsilon}, F_{r}^{\varepsilon}, F_{\theta}^{\varepsilon}\right):=\left(F_{1}^{\varepsilon}, F_{2}^{\varepsilon}, 0\right)$, this condition reads $\partial_{z}\left(r F_{z}^{\varepsilon}\right)+\partial_{r}\left(r F_{r}^{\varepsilon}\right)=0$, which is equivalent to assume that the vector field $\boldsymbol{F}^{\varepsilon}$, expressed in Cartesian coordinates, is divergence free.

(b) There exist $C_{F}, L>0$ such that, for any $\varepsilon \in(0,1)$ and $t \geq 0$,

$$
\left|F^{\varepsilon}(x, t)\right| \leq \frac{C_{F}}{|\log \varepsilon|}, \quad\left|F^{\varepsilon}(x, t)-F^{\varepsilon}(y, t)\right| \leq \frac{L}{|\log \varepsilon|}|x-y| \quad \forall x, y \in \mathbb{R}^{2} .
$$

Theorem 2.2. Under Assumption 2.1, let

$$
\zeta(t)=\zeta^{0}+\frac{a}{4 \pi r_{0}}\left(\begin{array}{l}
1 \\
0
\end{array}\right) t .
$$

Then, for each $T>0$ the following holds true.

(1) For any $k \in\left(0, \frac{1}{4}\right)$ there is $C_{k}>0$ such that, for any $\varepsilon$ small enough,

$$
\Lambda_{\varepsilon}(t):=\operatorname{supp} \omega_{\varepsilon}(\cdot, t) \subset\left\{x \in \mathbb{R}^{2}:\left|x_{2}-r_{0}\right| \leq C_{k}|\log \varepsilon|^{-k}\right\} \quad \forall t \in[0, T] .
$$

(2) For any $\varepsilon$ small enough there are $\zeta^{\varepsilon}(t) \in \Pi, t \in[0, T]$, and $\varrho_{\varepsilon}>0$ such that

$$
\lim _{\varepsilon \rightarrow 0}|\log \varepsilon| \int_{\Sigma\left(\zeta^{\varepsilon}(t) \mid \varrho_{\varepsilon}\right)} \mathrm{d} x \omega_{\varepsilon}(x, t)=a,
$$

with

$$
\lim _{\varepsilon \rightarrow 0} \varrho_{\varepsilon}=0, \quad \lim _{\varepsilon \rightarrow 0} \zeta^{\varepsilon}(t)=\zeta(t)
$$

\subsection{Proof of Theorem 1.1}

Given $T$ as in the statement of the theorem, we fix $R<D$ and let

$$
T_{\varepsilon}:=\max \left\{t \in[0, T]:\left|x_{2}-r_{j}\right| \leq R \forall x \in \Lambda_{j, \varepsilon}(s) \forall s \in[0, t] \forall j=1, \ldots, N\right\} .
$$

By continuity, from Eqs. (1.10) and (1.11) it follows that $T_{\varepsilon}>0$ for any $\varepsilon$ sufficiently small. Moreover, in view of Eq. (1.12), for any $t \in\left[0, T_{\varepsilon}\right]$ the rings evolve with supports $\Lambda_{i, \varepsilon}(t)$ that remain separated from each other by a distance larger than or equal to $2(D-R)$. Now, in view of Eqs. (1.13) and (1.14), the $i$-th vortex ring $\omega_{i, \varepsilon}(x, t)$ satisfies

$$
\omega_{i, \varepsilon}(x(t), t)=\frac{x_{2}(t)}{x_{2}(0)} \omega_{i, \varepsilon}(x(0), 0),
$$

where $x(t)$ is solution to

$$
\dot{x}(t)=u_{i}(x(t), t)+G^{i, \varepsilon}(x(t), t), \quad G^{i, \varepsilon}(x, t):=\sum_{j: j \neq i} u_{j}(x, t),
$$

with

$$
u_{j}(x, t):=\int \mathrm{d} y H(x, y) \omega_{j, \varepsilon}(y, t) \quad j=1, \ldots, N .
$$

Since the integration in the right-hand side of Eq. (2.15) is restricted to the support $\Lambda_{j, \varepsilon}(t)$, for each $t \in\left[0, T_{\varepsilon}\right]$ the field $G^{i, \varepsilon}(x, t)$ in Eq. (2.14) is regular in the set $\Lambda_{i, \varepsilon}(t)$. More precisely, in Appendix B we prove the following lemma.

Lemma 2.3. There exist vector fields $F^{i, \varepsilon}=\left(F_{1}^{i, \varepsilon}, F_{2}^{i, \varepsilon}\right), i=1, \ldots, N$, such that 
(i) $\partial_{x_{1}}\left(x_{2} F_{1}^{i, \varepsilon}\right)+\partial_{x_{2}}\left(x_{2} F_{2}^{i, \varepsilon}\right)=0$;

(ii) for some constant $\bar{C}>0$, any $i=1, \ldots, N$, and $t \in\left[0, T_{\varepsilon}\right]$,

$$
\left|F^{i, \varepsilon}(x, t)\right| \leq \frac{\bar{C}}{|\log \varepsilon|}, \quad\left|F^{i, \varepsilon}(x, t)-F^{i, \varepsilon}(y, t)\right| \leq \frac{\bar{C}}{|\log \varepsilon|}|x-y| \quad \forall x, y \in \mathbb{R}^{2} ;
$$

(iii) $F^{i, \varepsilon}(x, t)=G^{i, \varepsilon}(x, t)$ for any $x \in \Lambda_{i, \varepsilon}(t)$ and $t \in\left[0, T_{\varepsilon}\right]$.

Therefore, as the characteristics involved in Eq. (2.13) satisfy $x(t) \in \Lambda_{i, \varepsilon}(t)$, they solve $\dot{x}(t)=$ $u_{i}(x(t), t)+F^{i, \varepsilon}(x(t), t)$ for any $t \in\left[0, T_{\varepsilon}\right]$. Hence, we can apply Theorem 2.2 to the evolution of the $i$-th vortex ring, with parameters $\left(a_{i}, \zeta^{i}, T, k\right)$ in place of $\left(a, \zeta^{0}, T, k\right)$, and conclude that, for any $\varepsilon$ small enough,

(1) $\left|x_{2}-r_{i}\right| \leq C_{k}|\log \varepsilon|^{-k}$ for any $x \in \Lambda_{i, \varepsilon}(t), t \in\left[0, T_{\varepsilon}\right]$, and $i=1, \ldots, N$,

(2) there are $\zeta^{i, \varepsilon}(t) \in \Pi, i=1, \ldots, N$, and $\varrho_{\varepsilon}>0$ such that

$$
\lim _{\varepsilon \rightarrow 0}|\log \varepsilon| \int_{\Sigma\left(\zeta^{i, \varepsilon}(t) \mid \varrho_{\varepsilon}\right)} \mathrm{d} x \omega_{\varepsilon}(x, t)=a_{i},
$$

with

$$
\lim _{\varepsilon \rightarrow 0} \varrho_{\varepsilon}=0, \quad \lim _{\varepsilon \rightarrow 0} \zeta^{i, \varepsilon}(t)=\zeta^{i}(t) .
$$

In particular, (1) implies that $\left|x_{2}-r_{i}\right| \leq R / 2$ for any $x \in \Lambda_{i, \varepsilon}(t), t \in\left[0, T_{\varepsilon}\right]$, and $i=1, \ldots, N$, definitively for $\varepsilon$ small enough. Therefore, by continuity, $T_{\varepsilon}=T$ for any $\varepsilon$ small enough, and Theorem 1.1 is thus proved.

\section{The reduced system: analysis of the radial motion}

The proof Theorem 2.2 is split in two parts. The first one, which is the content of the present section, concerns the sharp localization property of the vorticity along the radial direction as stated in item (1) of Theorem 2.2. Without loss of generality, we consider the case $a=1$; hence, Eq. (2.8) reads

$$
0 \leq \omega_{\varepsilon}(x, 0) \leq \frac{M}{\varepsilon^{2}|\log \varepsilon|} \quad \forall x \in \mathbb{R}^{2}, \quad|\log \varepsilon| \int \mathrm{d} y \omega_{\varepsilon}(y, 0)=1 .
$$

The following weak formulation will be used, which is a direct generalization of Eq. (1.9),

$$
\frac{\mathrm{d}}{\mathrm{d} t} \int \mathrm{d} x \omega_{\varepsilon}(x, t) f(x, t)=\int \mathrm{d} x \omega_{\varepsilon}(x, t)\left[\left(u+F^{\varepsilon}\right) \cdot \nabla f+\partial_{t} f\right](x, t),
$$

where $f=f(x, t)$ is any bounded smooth test function. Moreover, the kernel $H(x, y)$ in Eq. (2.2) can be split as made in [5, Lemma 3.3], where it is shown that the most singular part of $H(x, y)$ is given by the kernel $K(x-y)$ corresponding to the planar case,

$$
K(x)=\nabla^{\perp} G(x), \quad G(x):=-\frac{1}{2 \pi} \log |x|,
$$

where $v^{\perp}:=\left(v_{2},-v_{1}\right)$ for $v=\left(v_{1}, v_{2}\right)$. More precisely, for any $x, y \in \Pi$,

$$
H(x, y)=K(x-y)+L(x, y)+\mathcal{R}(x, y),
$$

where

$$
L(x, y)=\frac{1}{4 \pi x_{2}} \log \frac{1+|x-y|}{|x-y|}\left(\begin{array}{l}
1 \\
0
\end{array}\right)
$$


and there exists $C_{0}>0$ such that, for any $x, y \in \Pi$,

$$
|\mathcal{R}(x, y)| \leq C_{0} \frac{1+x_{2}+\sqrt{x_{2} y_{2}}\left(1+\left|\log \left(x_{2} y_{2}\right)\right|\right)}{x_{2}^{2}} .
$$

A notation warning: In what follows, we shall denote by $C$ a generic positive constant, whose numerical value may change from line to line and it may possibly depend on the parameters $\zeta^{0}=\left(z_{0}, r_{0}\right)$ and $M$ appearing in Theorem 2.2 and Eq. (3.1), as well as on the given time $T$.

As claimed at the beginning of the section, our goal is to show that, under Assumption 2.1, for any $T>0$ and $k \in\left(0, \frac{1}{4}\right)$, if $\varepsilon$ is small enough then

$$
\left|x_{2}-r_{0}\right| \leq \frac{C}{|\log \varepsilon|^{k}} \quad \forall x \in \Lambda_{\varepsilon}(t) \quad \forall t \in[0, T] .
$$

We let

$$
T_{\varepsilon}^{0}:=\max \left\{t \in[0, T]: \frac{r_{0}}{2} \leq x_{2} \leq \frac{3}{2} r_{0} \quad \forall x \in \Lambda_{\varepsilon}(s) \forall s \in[0, t]\right\}
$$

and assume hereafter $\varepsilon<r_{0} / 2$ so that $T_{\varepsilon}^{0}>0$ in view of Eq. (2.9). In what follows, we show that, for any $k \in\left(0, \frac{1}{4}\right)$,

$$
\left|x_{2}-r_{0}\right| \leq \frac{C}{|\log \varepsilon|^{k}} \quad \forall x \in \Lambda_{\varepsilon}(t) \quad \forall t \in\left[0, T_{\varepsilon}^{0}\right]
$$

provided $\varepsilon$ is small enough. By continuity, this implies that $T_{\varepsilon}^{0}=T$ (for $\varepsilon$ sufficiently small), from which Eq. (3.7) follows for $\varepsilon$ sufficiently small.

The proof of Eq. (3.8) is quite long, so it is divided into three preliminary lemmas plus a conclusion. Preliminarily, it is useful to decompose the velocity field according to Eq. (3.4), writing

$$
u(x, t)=\widetilde{u}(x, t)+\int \mathrm{d} y L(x, y) \omega_{\varepsilon}(y, t)+\int \mathrm{d} y \mathcal{R}(x, y) \omega_{\varepsilon}(y, t),
$$

where $\widetilde{u}(x, t)=\int \mathrm{d} y K(x-y) \omega_{\varepsilon}(y, t)$.

Lemma 3.1. The following estimates hold true,

$$
\int \mathrm{d} y|L(x, y)| \omega_{\varepsilon}(y, t) \leq C, \quad \int \mathrm{d} y|\mathcal{R}(x, y)| \omega_{\varepsilon}(y, t) \leq \frac{C}{|\log \varepsilon|} \quad \forall t \in\left[0, T_{\varepsilon}^{0}\right] .
$$

Proof. From Eqs. (3.5) and (3.6), it follows that

$$
|L(x, y)| \leq \frac{1}{2 \pi r_{0}} \log \frac{1+|x-y|}{|x-y|}, \quad|\mathcal{R}(x, y)| \leq C \quad \forall x, y \in \Lambda_{\varepsilon}(t) \quad \forall t \in\left[0, T_{\varepsilon}^{0}\right],
$$

while, from Eqs. (2.3), (3.1), and the definition of $T_{\varepsilon}^{0}$,

$$
\left|\omega_{\varepsilon}(x, t)\right| \leq \frac{3 M}{\varepsilon^{2}|\log \varepsilon|} \quad \forall t \in\left[0, T_{\varepsilon}^{0}\right] .
$$

Since $\log \frac{1+|x-y|}{|x-y|}$ is monotonically unbounded as $y \rightarrow x$, the maximum of $\int \mathrm{d} y \log \frac{1+|x-y|}{|x-y|} \omega_{\varepsilon}(y, t)$ is achieved when we rearrange the vorticity mass as close as possible to the singularity. Therefore, in view 
of Eq. (3.12),

$$
\begin{aligned}
\int \mathrm{d} y|L(x, y)| \omega_{\varepsilon}(y, t) & \leq \frac{1}{2 \pi r_{0}} \int \mathrm{d} y \log \frac{1+|x-y|}{|x-y|} \omega_{\varepsilon}(y, t) \\
& \leq \frac{3 M}{\varepsilon^{2}|\log \varepsilon| r_{0}} \int_{0}^{\bar{\rho}} \mathrm{d} \rho \rho \log \frac{1+\rho}{\rho} \\
& =\frac{3 M}{\varepsilon^{2}|\log \varepsilon| r_{0}}\left\{\frac{\bar{\rho}^{2}}{2} \log \frac{1+\bar{\rho}}{\bar{\rho}}+\frac{1}{2} \int_{0}^{\bar{\rho}} \mathrm{d} \rho \frac{\rho}{1+\rho}\right\},
\end{aligned}
$$

with $\bar{\rho}$ such that $3 \pi \bar{\rho}^{2} M /\left(\varepsilon^{2}|\log \varepsilon|\right)=1 /|\log \varepsilon|$, from which the first estimate in Eq. (3.10) follows. Finally, we observe that, by Liouville's theorem and Eq. (2.3), with $\boldsymbol{F}^{\varepsilon}$ as in Assumption 2.1-(a),

$$
\int \mathrm{d} y \omega_{\varepsilon}(y, t)=\frac{1}{2 \pi} \int \mathrm{d} \boldsymbol{\xi} \frac{\omega_{\varepsilon}(\boldsymbol{\xi}, t)}{r}=\frac{1}{2 \pi} \int \mathrm{d} \boldsymbol{\xi}_{0} \frac{\omega_{\varepsilon}\left(\boldsymbol{\xi}_{0}, 0\right)}{r_{0}}=\int \mathrm{d} y \omega_{\varepsilon}(y, 0)=\frac{1}{|\log \varepsilon|},
$$

where we have used the coordinate transformation $\boldsymbol{\xi}=\phi^{t}\left(\boldsymbol{\xi}_{0}\right)$, with $\phi^{t}$ the flow generated by $\dot{\boldsymbol{\xi}}=$ $\boldsymbol{u}(\boldsymbol{\xi}, t)+\boldsymbol{F}^{\varepsilon}(\boldsymbol{\xi}, t)$. Therefore, the second estimate in Eq. (3.10) is a consequence of the second one in Eq. (3.11).

We denote by $B_{\varepsilon}(t)=\left(B_{\varepsilon, 1}(t), B_{\varepsilon, 2}(t)\right)$ the center of vorticity of the blob, defined by

$$
B_{\varepsilon}(t)=\frac{\int \mathrm{d} x x \omega_{\varepsilon}(x, t)}{\int \mathrm{d} x \omega_{\varepsilon}(x, t)}=|\log \varepsilon| \int \mathrm{d} x x \omega_{\varepsilon}(x, t),
$$

and by $I_{\varepsilon}(t)$ the axial moment of inertia with respect to $x_{2}=B_{\varepsilon, 2}(t)$, i.e.,

$$
I_{\varepsilon}(t)=\int \mathrm{d} x\left(x_{2}-B_{\varepsilon, 2}(t)\right)^{2} \omega_{\varepsilon}(x, t) .
$$

Since $\Lambda_{\varepsilon}(t)$ is compact, the time derivatives of $B_{\varepsilon, 2}(t)$ (in this section, we are only interested in this component) and $I_{\varepsilon}(t)$ can be computed by means of Eq. (3.2). To this end, we first observe that the time derivative of $M_{2}:=\int \mathrm{d} x \omega(x, t) x_{2}^{2}$ is $\dot{M}_{2}=\int \mathrm{d} x \omega_{\varepsilon}(x, t) 2 x_{2} F_{2}^{\varepsilon}(x, t)$ (it is a conserved quantity in absence of external field, see Appendix A), so that

$$
\begin{aligned}
& \dot{B}_{\varepsilon, 2}(t)=|\log \varepsilon| \int \mathrm{d} x \omega_{\varepsilon}(x, t)\left(F_{2}^{\varepsilon}(x, t)+\int \mathrm{d} y \mathcal{R}_{2}(x, y) \omega_{\varepsilon}(y, t)\right), \\
& \dot{I}_{\varepsilon}(t)=2 \int \mathrm{d} x \omega_{\varepsilon}(x, t)\left(x_{2}-B_{\varepsilon, 2}(t)\right) F_{2}^{\varepsilon}(x, t)-2 B_{\varepsilon, 2}(t) \int \mathrm{d} x \omega_{\varepsilon}(x, t) \int \mathrm{d} y \mathcal{R}_{2}(x, y) \omega_{\varepsilon}(y, t),
\end{aligned}
$$

where we have used the expression Eq. (3.9) for $u(x, t)$, the identities

$$
\int \mathrm{d} x \omega_{\varepsilon}(x, t)\left(x_{2}-B_{\varepsilon, 2}(t)\right)=0, \quad \int \mathrm{d} x \widetilde{u}(x, t) \omega_{\varepsilon}(x, t)=0,
$$

[which derive from the definition of center of vorticity and the explicit form of $K(x-y)$ in Eq. (3.3)], and the fact that $L_{2}(x, y)=0$, see Eq. (3.5).

Lemma 3.2. The following estimate holds,

$$
I_{\varepsilon}(t) \leq \frac{C}{|\log \varepsilon|^{2}} \quad \forall t \in\left[0, T_{\varepsilon}^{0}\right]
$$

Proof. By Eqs. (2.10), (3.10), (3.17), and (3.18), we have that, for any $\left[0, T_{\varepsilon}^{0}\right],\left|\dot{B}_{\varepsilon, 2}(t)\right| \leq C /|\log \varepsilon|$ (hence $\left.\left|B_{\varepsilon, 2}(t)\right| \leq C\right)$ and

$$
\left|\dot{I}_{\varepsilon}(t)\right| \leq \frac{C}{|\log \varepsilon|} \int \mathrm{d} x\left|x_{2}-B_{\varepsilon, 2}(t)\right| \omega_{\varepsilon}(x, t)+\frac{C}{|\log \varepsilon|^{2}} \leq \frac{C}{|\log \varepsilon|^{3 / 2}} \sqrt{I_{\varepsilon}(t)}+\frac{C}{|\log \varepsilon|^{2}},
$$


where in the last estimate we used the Cauchy-Schwarz inequality and again Eq. (3.14). Eq. (3.19) now follows by integration of the last differential inequality since the initial data imply $I_{\varepsilon}(0) \leq 4 \varepsilon^{2}$.

We remark that the estimate Eq. (3.19) is sufficient for our purposes but we do not know whether it is optimal. In [5], a more sophisticated argument allows to obtain the same estimate $C /|\log \varepsilon|^{2}$ for the moment of inertia with respect to the center of vorticity, but the whole argument works only for short times. In our case, we are able to extend the analysis to any positive time not only because of the estimate Eq. (3.19) for the axial moment of inertia, but above all for the absence of the non-Lipschitz term Eq. (3.5) when we analyze separately the motion along the radial direction in Lemmata 3.3 and 3.4, see Remark 3.1 below. After that, we will need and prove a weaker estimate $C /|\log \varepsilon|$ (global in time) for the moment of inertia with respect to the center of vorticity in order to conclude the analysis of the whole motion.

Lemma 3.3. Recall $\Lambda_{\varepsilon}(t)=\operatorname{supp} \omega_{\varepsilon}(\cdot, t)$ and define

$$
R_{t}:=\max \left\{\left|x_{2}-B_{\varepsilon, 2}(t)\right|: x \in \Lambda_{\varepsilon}(t)\right\} .
$$

Given $x_{0} \in \Lambda_{\varepsilon}(0)$, let $x\left(x_{0}, t\right)$ be the solution to Eq. (2.7) with initial condition $x\left(x_{0}, 0\right)=x_{0}$ and suppose at time $t \in\left(0, T_{\varepsilon}^{0}\right]$, it happens that

$$
\left|x_{2}\left(x_{0}, t\right)-B_{\varepsilon, 2}(t)\right|=R_{t} .
$$

Then, at this time $t$,

$$
\frac{\mathrm{d}}{\mathrm{d} t}\left|x_{2}\left(x_{0}, t\right)-B_{\varepsilon, 2}(t)\right| \leq \frac{C}{|\log \varepsilon|}+\frac{1}{\pi R_{t}|\log \varepsilon|}+\sqrt{\frac{C m_{t}\left(R_{t} / 2\right)}{\varepsilon^{2}|\log \varepsilon|}},
$$

where the function $m_{t}(\cdot)$ is defined by

$$
m_{t}(h)=\int_{\left|y_{2}-B_{\varepsilon, 2}(t)\right|>h} \mathrm{~d} y \omega_{\varepsilon}(y, t) .
$$

Proof. We observe that the proof is similar to that given in [4, Lemma 2.5]. Letting $x=x\left(x_{0}, t\right)$, by Eqs. (2.7), (3.9), (3.14), and (3.17) we have,

$$
\begin{aligned}
\frac{\mathrm{d}}{\mathrm{d} t}\left|x_{2}\left(x_{0}, t\right)-B_{\varepsilon, 2}(t)\right| & =\left(u_{2}(x, t)+F_{2}^{\varepsilon}(x, t)-\dot{B}_{\varepsilon, 2}(t)\right) \frac{x_{2}-B_{\varepsilon, 2}(t)}{\left|x_{2}-B_{\varepsilon, 2}(t)\right|} \\
& =V(x, t) \frac{x_{2}-B_{\varepsilon, 2}(t)}{\left|x_{2}-B_{\varepsilon, 2}(t)\right|}+\int \mathrm{d} y K_{2}(x-y) \omega_{\varepsilon}(y, t) \frac{x_{2}-B_{\varepsilon, 2}(t)}{\left|x_{2}-B_{\varepsilon, 2}(t)\right|},
\end{aligned}
$$

with

$$
\begin{aligned}
V(x, t)= & F_{2}^{\varepsilon}(x, t)+\int \mathrm{d} z \mathcal{R}_{2}(x, z) \omega_{\varepsilon}(z, t) \\
& -|\log \varepsilon| \int \mathrm{d} y \omega_{\varepsilon}(y, t)\left(F_{2}^{\varepsilon}(y, t)+\int \mathrm{d} z \mathcal{R}_{2}(y, z) \omega_{\varepsilon}(z, t)\right) .
\end{aligned}
$$

From Eqs. (2.10), (3.10), and (3.14), we have

$$
|V(x, t)| \leq \frac{C}{|\log \varepsilon|} \quad \forall t \in\left[0, T_{\varepsilon}^{0}\right] .
$$

For the last term in Eq. (3.24), we split the integration region into two parts, the set $A_{1}=\{y \in$ $\left.\Lambda_{\varepsilon}(t):\left|y_{2}-B_{\varepsilon, 2}(t)\right| \leq R_{t} / 2\right\}$ and the set $A_{2}=\left\{y \in \Lambda_{\varepsilon}(t): R_{t} / 2<\left|y_{2}-B_{\varepsilon, 2}(t)\right| \leq R_{t}\right\}$. Then,

$$
\int \mathrm{d} y K_{2}(x-y) \omega_{\varepsilon}(y, t) \frac{x_{2}-B_{\varepsilon, 2}(t)}{\left|x_{2}-B_{\varepsilon, 2}(t)\right|}=H_{1}+H_{2}
$$


where

$$
H_{1}=\frac{x_{2}-B_{\varepsilon, 2}(t)}{\left|x_{2}-B_{\varepsilon, 2}(t)\right|} \int_{A_{1}} \mathrm{~d} y K_{2}(x-y) \omega_{\varepsilon}(y, t)
$$

and

$$
H_{2}=\frac{x_{2}-B_{\varepsilon, 2}(t)}{\left|x_{2}-B_{\varepsilon, 2}(t)\right|} \int_{A_{2}} \mathrm{~d} y K_{2}(x-y) \omega_{\varepsilon}(y, t) .
$$

We consider first the contribution due to the set $A_{1}$. Recalling Eq. (3.3), after introducing the new variables $x^{\prime}=x-B_{\varepsilon}(t), y^{\prime}=y-B_{\varepsilon}(t)$, we get,

$$
\left|H_{1}\right| \leq \frac{1}{2 \pi} \int_{\left|y_{2}^{\prime}\right| \leq R_{t} / 2} \mathrm{~d} y^{\prime} \frac{1}{\left|x^{\prime}-y^{\prime}\right|} \omega_{\varepsilon}\left(y^{\prime}+B_{\varepsilon}(t)\right) .
$$

From Eq. (3.21) we have $\left|x_{2}^{\prime}\right|=R_{t}$, and hence $\left|y_{2}^{\prime}\right| \leq R_{t} / 2$ implies $\left|x^{\prime}-y^{\prime}\right| \geq\left|x_{2}^{\prime}-y_{2}^{\prime}\right| \geq R_{t} / 2$, so that

$$
\left|H_{1}\right| \leq \frac{1}{\pi R_{t}} \int_{\left|y_{2}^{\prime}\right| \leq R_{t} / 2} \mathrm{~d} y^{\prime} \omega_{\varepsilon}\left(y^{\prime}+B_{\varepsilon}(t)\right) \leq \frac{1}{\pi R_{t}|\log \varepsilon|} .
$$

We bound now $H_{2}$. Again by Eq. (3.3),

$$
\left|H_{2}\right| \leq \frac{1}{2 \pi} \int_{A_{2}} \mathrm{~d} y \frac{1}{|x-y|} \omega_{\varepsilon}(y, t) .
$$

The function $|x-y|^{-1}$ diverges monotonically as $y \rightarrow x$, and so the maximum of the integral is obtained when we rearrange the vorticity mass as close as possible to the singularity. By Eq. (3.12) and since, by Eq. (3.23), $m_{t}\left(R_{t} / 2\right)$ is equal to the total amount of vorticity in $A_{2}$, this rearrangement gives,

$$
\left|H_{2}\right| \leq \frac{3 M \varepsilon^{-2}}{2 \pi|\log \varepsilon|} \int_{\Sigma(0 \mid r)} \mathrm{d} y^{\prime} \frac{1}{\left|y^{\prime}\right|}=\frac{3 M \varepsilon^{-2}}{|\log \varepsilon|} r,
$$

where the radius $r$ is such that $3 \pi r^{2} M /\left(\varepsilon^{2}|\log \varepsilon|\right)=m_{t}\left(R_{t} / 2\right)$. The estimate Eq. (3.22) now follows by Eqs. (3.24), (3.26), (3.27), (3.31), and (3.32).

We determine now the behavior of the function $m_{t}(\cdot)$ introduced in Eq. (3.23) when its argument goes to 0 . The proof will be adapted from that of [5, Proposition 3.4].

Lemma 3.4. Let $m_{t}$ be defined as in Eq. (3.23). For each $\ell>0$ and $k \in\left(0, \frac{1}{4}\right)$,

$$
\lim _{\varepsilon \rightarrow 0} \max _{t \in\left[0, T_{\varepsilon}^{0}\right]} \varepsilon^{-\ell} m_{t}\left(\frac{1}{|\log \varepsilon|^{k}}\right)=0 .
$$

Proof. Given $R \geq 2 h^{\alpha}, h>0$, and $\alpha$ a positive parameter to be fixed later, let $W_{R, h}\left(x_{2}\right)$, with $x_{2}$ the second component of $x=\left(x_{1}, x_{2}\right)$, be a nonnegative smooth function, such that

$$
W_{R, h}\left(x_{2}\right)= \begin{cases}1 & \text { if }\left|x_{2}\right| \leq R \\ 0 & \text { if }\left|x_{2}\right| \geq R+h\end{cases}
$$

and its derivative $W_{R, h}^{\prime}$ satisfies

$$
\begin{aligned}
& \left|W_{R, h}^{\prime}\left(x_{2}\right)\right|<\frac{C}{h} \\
& \left|W_{R, h}^{\prime}\left(x_{2}\right)-W_{R, h}^{\prime}\left(y_{2}\right)\right|<\frac{C}{h^{2}}\left|x_{2}-y_{2}\right| \leq \frac{C}{h^{2}}|x-y| .
\end{aligned}
$$


We introduce the quantity

$$
\mu_{t}(R, h)=\int \mathrm{d} x\left[1-W_{R, h}\left(x_{2}-B_{\varepsilon, 2}(t)\right)\right] \omega_{\varepsilon}(x, t),
$$

which is a mollified version of $m_{t}$ satisfying

$$
\mu_{t}(R, h) \leq m_{t}(R) \leq \mu_{t}(R-h, h) .
$$

Hence it is sufficient to prove (3.33) with $\mu_{t}$ in place of $m_{t}$. Since the function $t \mapsto \mu_{t}(R, h)$ is differentiable, we can compute its time derivative, by Eq. (3.2) with test function $f(x, t)=1-W_{R, h}\left(x_{2}-B_{\varepsilon, 2}(t)\right)$ and then using Eqs. (3.9) and (3.17). We have,

$$
\begin{aligned}
\frac{\mathrm{d}}{\mathrm{d} t} \mu_{t}(R, h) & =-\int \mathrm{d} x \nabla W_{R, h}\left(x_{2}-B_{\varepsilon, 2}(t)\right) \cdot\left[u(x, t)+F^{\varepsilon}(x, t)-\dot{B}_{\varepsilon}(t)\right] \omega_{\varepsilon}(x, t) \\
& =-\int \mathrm{d} x W_{R, h}^{\prime}\left(x_{2}-B_{\varepsilon, 2}(t)\right)\left[u_{2}(x, t)+F_{2}^{\varepsilon}(x, t)-\dot{B}_{\varepsilon, 2}(t)\right] \omega_{\varepsilon}(x, t) \\
& =-H_{3}-H_{4},
\end{aligned}
$$

with

$$
\begin{aligned}
H_{3} & =\int \mathrm{d} x W_{R, h}^{\prime}\left(x_{2}-B_{\varepsilon, 2}(t)\right) \int \mathrm{d} y K_{2}(x-y) \omega_{\varepsilon}(y, t) \omega_{\varepsilon}(x, t) \\
& =\frac{1}{2} \int \mathrm{d} x \int \mathrm{d} y \omega_{\varepsilon}(x, t) \omega_{\varepsilon}(y, t)\left[W_{R, h}^{\prime}\left(x_{2}-B_{\varepsilon, 2}(t)\right)-W_{R, h}^{\prime}\left(y_{2}-B_{\varepsilon, 2}(t)\right)\right] K_{2}(x-y), \\
H_{4} & =\int \mathrm{d} x W_{R, h}^{\prime}\left(x_{2}-B_{\varepsilon, 2}(t)\right) \omega_{\varepsilon}(x, t) V(x, t),
\end{aligned}
$$

where the antisymmetry of $K$ has allowed to achieve the second expression of $H_{3}$, and $V(x, t)$ is defined in Eq. (3.25). We can note that, in view of Eq. (3.26), (3.35), and the fact that $W_{R, h}^{\prime}(z)$ is zero if $|z| \leq R$,

$$
\left|H_{4}\right| \leq \frac{C}{h|\log \varepsilon|} m_{t}(R) \quad \forall t \in\left[0, T_{\varepsilon}^{0}\right]
$$

Now we treat $H_{3}$. We introduce the new variables $x^{\prime}=x-B_{\varepsilon}(t), y^{\prime}=y-B_{\varepsilon}(t)$ (as done previously), define $\widetilde{\omega}_{\varepsilon}(z, t):=\omega_{\varepsilon}\left(z+B_{\varepsilon}(t), t\right)$, and

$$
f\left(x^{\prime}, y^{\prime}\right):=\frac{1}{2} \widetilde{\omega}_{\varepsilon}\left(x^{\prime}, t\right) \widetilde{\omega}_{\varepsilon}\left(y^{\prime}, t\right)\left[W_{R, h}^{\prime}\left(x_{2}^{\prime}\right)-W_{R, h}^{\prime}\left(y_{2}^{\prime}\right)\right] K_{2}\left(x^{\prime}-y^{\prime}\right)
$$

whence $H_{3}=\int \mathrm{d} x^{\prime} \int \mathrm{d} y^{\prime} f\left(x^{\prime}, y^{\prime}\right)$. We note that $f\left(x^{\prime}, y^{\prime}\right)$ is a symmetric function of $x^{\prime}$ and $y^{\prime}$ and that, by Eq. (3.34), in order to be different from zero it is necessary that either $\left|x_{2}^{\prime}\right| \geq R$ or $\left|y_{2}^{\prime}\right| \geq R$. Therefore,

$$
\begin{aligned}
H_{3} & =\left[\int_{\left\lfloor x_{2}^{\prime} \mid>R\right.} \mathrm{d} x^{\prime} \int \mathrm{d} y^{\prime}+\int \mathrm{d} x^{\prime} \int_{\left|y_{2}^{\prime}\right|>R} \mathrm{~d} y^{\prime}-\int_{\left|x_{2}^{\prime}\right|>R} \mathrm{~d} x^{\prime} \int_{\left|y_{2}^{\prime}\right|>R} \mathrm{~d} y^{\prime}\right] f\left(x^{\prime}, y^{\prime}\right) \\
& =2 \int_{\left|x_{2}^{\prime}\right|>R} \mathrm{~d} x^{\prime} \int \mathrm{d} y_{2}^{\prime} f\left(x^{\prime}, y^{\prime}\right)-\int_{\left|x_{2}^{\prime}\right|>R} \mathrm{~d} x^{\prime} \int_{\left|y_{2}^{\prime}\right|>R} \mathrm{~d} y^{\prime} f\left(x^{\prime}, y^{\prime}\right) \\
& =H_{3}^{\prime}+H_{3}^{\prime \prime}+H_{3}^{\prime \prime \prime},
\end{aligned}
$$

with

$$
\begin{aligned}
& H_{3}^{\prime}=2 \int_{\left|x_{2}^{\prime}\right|>R} \mathrm{~d} x^{\prime} \int_{\left|y_{2}^{\prime}\right| \leq R-h^{\alpha}} \mathrm{d} y^{\prime} f\left(x^{\prime}, y^{\prime}\right), \\
& H_{3}^{\prime \prime}=2 \int_{\left|x_{2}^{\prime}\right|>R} \mathrm{~d} x^{\prime} \int_{\left|y_{2}^{\prime}\right|>R-h^{\alpha}} \mathrm{d} y^{\prime} f\left(x^{\prime}, y^{\prime}\right),
\end{aligned}
$$




$$
H_{3}^{\prime \prime \prime}=-\int_{\left|x_{2}^{\prime}\right|>R} \mathrm{~d} x^{\prime} \int_{\left|y_{2}^{\prime}\right|>R} \mathrm{~d} y^{\prime} f\left(x^{\prime}, y^{\prime}\right) .
$$

By the properties of $W_{R, h}$, we have $W_{R, h}^{\prime}\left(y_{2}^{\prime}\right)=0$ for $\left|y_{2}^{\prime}\right| \leq R$. In particular, $W_{R, h}^{\prime}\left(y_{2}^{\prime}\right)=0$ for $\left|y_{2}^{\prime}\right| \leq$ $R-h^{\alpha}$, then

$$
H_{3}^{\prime}=\int_{\left|x_{2}^{\prime}\right|>R} \mathrm{~d} x^{\prime} \widetilde{\omega}_{\varepsilon}\left(x^{\prime}, t\right) W_{R, h}^{\prime}\left(x_{2}^{\prime}\right) \int_{\left|y_{2}^{\prime}\right| \leq R-h^{\alpha}} \mathrm{d} y^{\prime} K_{2}\left(x^{\prime}-y^{\prime}\right) \widetilde{\omega}_{\varepsilon}\left(y^{\prime}, t\right)
$$

and therefore, in view of Eq. (3.35),

$$
\left|H_{3}^{\prime}\right| \leq \frac{C}{h} m_{t}(R) \sup _{\left|x_{2}^{\prime}\right|>R}\left|A_{3}\left(x^{\prime}, t\right)\right|,
$$

with

$$
A_{3}\left(x^{\prime}, t\right)=\int_{\left|y_{2}^{\prime}\right| \leq R-h^{\alpha}} \mathrm{d} y^{\prime} K_{2}\left(x^{\prime}-y^{\prime}\right) \widetilde{\omega}_{\varepsilon}\left(y^{\prime}, t\right) .
$$

We note that if $\left|x_{2}^{\prime}\right|>R$ then $\left|y_{2}^{\prime}\right| \leq R-h^{\alpha}$ implies $\left|x^{\prime}-y^{\prime}\right| \geq\left|x_{2}^{\prime}-y_{2}^{\prime}\right| \geq h^{\alpha}$, hence

$$
\left|A_{3}\left(x^{\prime}, t\right)\right| \leq \frac{1}{2 \pi} \int_{\left|y_{2}^{\prime}\right| \leq R-h^{\alpha}} \mathrm{d} y^{\prime} \frac{\widetilde{\omega}_{\varepsilon}\left(y^{\prime}, t\right)}{\left|x^{\prime}-y^{\prime}\right|} \leq \frac{1}{2 \pi h^{\alpha}} \int_{\left|y_{2}^{\prime}\right| \leq R-h^{\alpha}} \mathrm{d} y^{\prime} \widetilde{\omega}_{\varepsilon}\left(y^{\prime}, t\right) \leq \frac{1}{2 \pi h^{\alpha}|\log \varepsilon|} .
$$

We then obtain, by Eq. (3.41),

$$
\left|H_{3}^{\prime}\right| \leq \frac{C}{h^{1+\alpha}|\log \varepsilon|} m_{t}(R) .
$$

From Eq. (3.36), as $R \geq 2 h^{\alpha}$ implies $R-h^{\alpha} \geq R / 2$,

$$
\begin{aligned}
\left|H_{3}^{\prime \prime}\right|+\left|H_{3}^{\prime \prime \prime}\right| & \leq \frac{C}{h^{2}} \int_{\left|x_{2}^{\prime}\right| \geq R} \mathrm{~d} x^{\prime} \int_{\left|y_{2}^{\prime}\right| \geq R-h^{\alpha}} \mathrm{d} y^{\prime} \widetilde{\omega}_{\varepsilon}\left(y^{\prime}, t\right) \widetilde{\omega}_{\varepsilon}\left(x^{\prime}, t\right) \\
& \leq \frac{C}{h^{2}} \int_{\left|x_{2}^{\prime}\right| \geq R} \mathrm{~d} x^{\prime} \int_{\left|y_{2}^{\prime}\right| \geq R / 2} \mathrm{~d} y^{\prime} \widetilde{\omega}_{\varepsilon}\left(y^{\prime}, t\right) \widetilde{\omega}_{\varepsilon}\left(x^{\prime}, t\right) \leq \frac{C I_{\varepsilon}(t)}{h^{2} R^{2}} m_{t}(R),
\end{aligned}
$$

where in the last bound we used Chebyshev's inequality. Finally, by Eq. (3.19),

$$
\left|H_{3}\right| \leq C\left(\frac{1}{h^{1+\alpha}|\log \varepsilon|}+\frac{1}{h^{2} R^{2}|\log \varepsilon|^{2}}\right) m_{t}(R) \quad \forall t \in\left[0, T_{\varepsilon}^{0}\right] .
$$

From estimates Eqs. (3.43) and (3.40), recalling Eq. (3.39), we get,

$$
\frac{\mathrm{d}}{\mathrm{d} t} \mu_{t}(R, h) \leq A_{\varepsilon}(R, h) m_{t}(R) \quad \forall t \in\left[0, T_{\varepsilon}^{0}\right] .
$$

where

$$
A_{\varepsilon}(R, h)=C\left(\frac{1}{h^{1+\alpha}|\log \varepsilon|}+\frac{1}{h^{2} R^{2}|\log \varepsilon|^{2}}+\frac{1}{h|\log \varepsilon|}\right) .
$$

Therefore, by Eqs. (3.38) and (3.44),

$$
\mu_{t}(R, h) \leq \mu_{0}(R, h)+A_{\varepsilon}(R, h) \int_{0}^{t} \mathrm{~d} s \mu_{s}(R-h, h) \quad \forall t \in\left[0, T_{\varepsilon}^{0}\right] .
$$


We assume now $\varepsilon$ sufficiently small, and we iterate the last inequality $n=\lfloor|\log \varepsilon|\rfloor$ times (denoting with $\lfloor a\rfloor$ the integer part of $a>0$ ), from

$$
R_{0}=\frac{1}{|\log \varepsilon|^{k}} \quad \text { to } \quad R_{n}=\frac{1}{2|\log \varepsilon|^{k}}
$$

where $R_{n}=R_{0}-n h$, and consequently

$$
h=\frac{1}{2 n|\log \varepsilon|^{k}} .
$$

In order to assure the condition $R \geq 2 h^{\alpha}$ stated at the beginning of Lemma 3.4 (under which Eq. (3.44) has been deduced) we have to choose consistently $\alpha$. We make the choice

$$
\alpha=\frac{1-k}{1+k}-\delta, \quad \delta \in\left(0, \frac{1-2 k}{1+k}\right),
$$

which implies

$$
h^{\alpha} \approx C\left(\frac{1}{|\log \varepsilon|^{1+k}}\right)^{\alpha}=C\left(\frac{1}{|\log \varepsilon|^{1+k}}\right)^{\frac{1-k}{1+k}-\delta}=\frac{C}{|\log \varepsilon|^{1-k-(1+k) \delta}},
$$

with $k<1-k-(1+k) \delta$ [by the second of (3.47)], and therefore, if $\varepsilon$ is small enough, $h^{\alpha} \ll R$, when $R$ is in the range established by (3.46). Moreover, the quantity $A_{\varepsilon}(R, h)$ is bounded by $C|\log \varepsilon|^{q}$ with $q<1$, in fact

$$
\begin{aligned}
\frac{1}{h^{1+\alpha}|\log \varepsilon|} & \leq C \frac{\left(|\log \varepsilon|^{k+1}\right)^{1+\alpha}}{|\log \varepsilon|} \leq C|\log \varepsilon|^{1-\delta(k+1)}, \\
\frac{1}{h^{2} R^{2}|\log \varepsilon|^{2}} & \leq C \frac{|\log \varepsilon|^{4 k+2}}{|\log \varepsilon|^{2}} \leq|\log \varepsilon|^{4 k} \\
\frac{1}{h|\log \varepsilon|} & \leq C|\log \varepsilon|^{k}
\end{aligned}
$$

In conclusion,

$$
\begin{aligned}
\mu_{t}\left(R_{0}-h, h\right) \leq & \mu_{0}\left(R_{0}-h, h\right)+\sum_{j=1}^{n-1} \mu_{0}\left(R_{j}, h\right) \frac{\left(C|\log \varepsilon|^{q} t\right)^{j}}{j !} \\
& +\frac{\left(C|\log \varepsilon|^{q}\right)^{n}}{(n-1) !} \int_{0}^{t} \mathrm{~d} s(t-s)^{n-1} \mu_{s}\left(R_{n}, h\right) \quad \forall t \in\left[0, T_{\varepsilon}^{0}\right] .
\end{aligned}
$$

Since $\Lambda_{\varepsilon}(0) \subset \Sigma(z \mid \varepsilon)$, we can determine $\varepsilon$ small enough so that $\mu_{0}\left(R_{j}, h\right)=0$ for any $j=0, \ldots, n$, hence, for any $t \in\left[0, T_{\varepsilon}^{0}\right]$,

$$
\mu_{t}\left(R_{0}-h, h\right) \leq \frac{\left(C|\log \varepsilon|^{q}\right)^{n}}{(n-1) !} \int_{0}^{t} \mathrm{~d} s(t-s)^{n-1} \mu_{s}\left(R_{n}, h\right) \leq \frac{\left(C|\log \varepsilon|^{q} t\right)^{n}}{n !},
$$

where in the last inequality we have used the trivial bound $\mu_{s}\left(R_{n}, h\right) \leq 1$. Therefore, using also Eq. (3.38), Stirling formula, and $n=\lfloor|\log \varepsilon|\rfloor$,

$$
m_{t}\left(R_{0}\right) \leq \mu_{t}\left(R_{0}-h, h\right) \leq \frac{C}{|\log \varepsilon|^{(1-q)|\log \varepsilon|}} \quad \forall t \in\left[0, T_{\varepsilon}^{0}\right]
$$

which implies Eq. (3.33). 
Remark 3.1. In [5, Prop. 3.4] a similar concentration result is deduced for the vorticity mass outside a small disk, but only for small times. This is due to the non-Lipschitz term Eq. (3.5) (not present in our case), which leads to an estimate like Eq. (3.48) but with $q=1$. We also remark that a weaker estimate $C /|\log \varepsilon|^{\theta}$ with $\theta \in(1,2)$ for the axial moment of inertia instead of Eq. (3.19) would lead as well to Eq. (3.48) (choosing $k \in\left(0, \frac{\theta-1}{4}\right)$ in this case).

Proof of Eq. (3.8). In view of Eqs. (2.10), (3.10), and (3.14), from (3.17) and since $\left|B_{\varepsilon, 2}(0)-r_{0}\right| \leq \varepsilon$ we have,

$$
\left|B_{\varepsilon, 2}(t)-r_{0}\right| \leq \frac{C}{|\log \varepsilon|} \quad \forall t \in\left[0, T_{\varepsilon}^{0}\right] .
$$

Therefore, it is sufficient to show that, given $k \in\left(0, \frac{1}{4}\right)$,

$$
\left|x_{2}-B_{\varepsilon, 2}(t)\right| \leq \frac{C}{|\log \varepsilon|^{k}} \quad \forall x \in \Lambda_{\varepsilon}(t) \quad \forall t \in\left[0, T_{\varepsilon}^{0}\right],
$$

provided $\varepsilon$ is small enough. To this end, we first notice that, in view of Lemma 3.3 and Eq. (3.20), for any $x_{0} \in \Lambda_{\varepsilon}(0)$ and $t \in\left[0, T_{\varepsilon}^{0}\right]$ we have $\left|x_{2}\left(x_{0}, t\right)-B_{\varepsilon, 2}(t)\right| \leq R_{t}$, and whenever $\left|x_{2}\left(x_{0}, t\right)-B_{\varepsilon, 2}(t)\right|=R_{t}$ the differential inequality Eq. (3.22) holds true. We claim that this implies

$$
\Lambda_{\varepsilon}(t) \subset\left\{x \in \mathbb{R}^{2}:\left|x_{2}-B_{\varepsilon, 2}(t)\right|<\rho(t)\right\} \quad \forall t \in\left[s_{0}, s_{1}\right] \quad \forall\left[s_{0}, s_{1}\right] \subseteq\left[0, T_{\varepsilon}^{0}\right],
$$

provided $\rho(t)$ solves

$$
\dot{\rho}(t)=\frac{2 C}{|\log \varepsilon|}+\frac{2}{\pi|\log \varepsilon| \rho(t)}+g(t),
$$

with initial datum $\rho\left(s_{0}\right)>R_{s_{0}}$ and $g(t)$ any smooth function which is an upper bound for the last term in Eq. (3.22). Indeed, $\left|x_{2}-B_{\varepsilon, 2}\left(s_{0}\right)\right|<\rho\left(s_{0}\right)$ for any $x \in \Lambda_{\varepsilon}\left(s_{0}\right)$ and, by absurd, if there were a first time $t_{*} \in\left(s_{0}, s_{1}\right]$ such that $\left|x_{2}\left(x_{0}, t_{*}\right)-B_{\varepsilon, 2}\left(t_{*}\right)\right|=\rho\left(t_{*}\right)$ for some $x_{0} \in \Lambda_{\varepsilon}(0)$, it would be necessarily $\rho\left(t_{*}\right)=R_{t_{*}}$; hence, by Eq. (3.22), $\dot{\rho}\left(t_{*}\right)$ would be strictly larger than $\frac{\mathrm{d}}{\mathrm{d} t}\left|x_{2}\left(x_{0}, t\right)-B_{\varepsilon, 2}(t)\right|_{t=t_{*}}$, which contradicts the characterization of $t_{*}$ as the first time at which the graph of $t \mapsto\left|x_{2}\left(x_{0}, t\right)-B_{\varepsilon, 2}(t)\right|$ crosses the one of $t \mapsto \rho(t)$.

Now, let

$$
t_{0}=\sup \left\{t \in\left[0, T_{\varepsilon}^{0}\right]: R_{s}<3|\log \varepsilon|^{-k} \forall s \in[0, t]\right\} .
$$

If $t_{0}=T_{\varepsilon}^{0}$ then Eq. (3.50) is already achieved, otherwise we set

$$
t_{1}=\sup \left\{t \in\left[t_{0}, T_{\varepsilon}^{0}\right]: R_{s}>2|\log \varepsilon|^{-k} \forall s \in\left[t_{0}, t_{1}\right]\right\}
$$

and consider $\rho(t)$ as in Eq. (3.52), relative to the interval $\left[s_{0}, s_{1}\right]=\left[t_{0}, t_{1}\right]$ and such that

$$
\rho\left(t_{0}\right)=4|\log \varepsilon|^{-k}, \quad g(t) \leq \frac{C \varepsilon^{(\ell-2) / 2}}{|\log \varepsilon|^{1 / 2}} \quad \forall t \in\left[t_{0}, t_{1}\right],
$$

for a fixed $\ell>2$. We note that $\rho\left(t_{0}\right)>R_{t_{0}}=3|\log \varepsilon|^{-k}$ and that, since $R_{t} \geq 2|\log \varepsilon|^{-k}$ for any $t \in\left[t_{0}, t_{1}\right]$, Eq. (3.33) guarantees that above condition on $g(t)$ is compatible with the requirement that the latter is an upper bound for the last term in Eq. (3.22).

Now, as $\rho(t) \geq R_{t} \geq 2|\log \varepsilon|^{-k}$ for any $t \in\left[t_{0}, t_{1}\right]$, the second term in the right-hand side of Eq. (3.52) is bounded by $C|\log \varepsilon|^{k-1}$, and therefore, since $k \in\left(0, \frac{1}{4}\right)$, from Eq. (3.52) we deduce that

$$
\dot{\rho}(t) \leq \frac{C}{|\log \varepsilon|^{3 / 4}} \quad \forall t \in\left[t_{0}, t_{1}\right]
$$

which integrated from $t_{0}$ and $t_{1}$ gives

$$
\rho(t) \leq \rho\left(t_{0}\right)+\frac{C T}{|\log \varepsilon|^{3 / 4}} \leq \frac{C}{|\log \varepsilon|^{k}} \quad \forall t \in\left[t_{0}, t_{1}\right] .
$$


Clearly, if $t_{1}=T_{\varepsilon}^{0}$ we are done. Otherwise, we can repeat the same argument in the intervals $\left[t_{0}^{\prime}, t_{1}^{\prime}\right] \subseteq$ $\left[t_{1}, T_{\varepsilon}^{0}\right]$ defined analogously to $\left[t_{0}, t_{1}\right]$ (if any). Eq. (3.50) is thus proved.

\section{The reduced system: analysis of the axial motion}

In this section we prove item (2) of Theorem 2.2, remarking that, as in the previous section, we always assume $a=1$. We premise a concentration result which shows that large part of the vorticity remains confined in a disk whose size is infinitesimal as $\varepsilon \rightarrow 0$.

Lemma 4.1. Consider the reduced system defined by Eqs. (2.2), (2.3), and (2.7). Under Assumption 2.1, with Eq. (3.1) in place of Eq. (2.8), for each $T>0$ there are $\varepsilon_{1} \in(0,1), C_{1}>0$ and $q_{\varepsilon}(t) \in \mathbb{R}^{2}$, such that

$$
|\log \varepsilon| \int_{\Sigma\left(q_{\varepsilon}(t), \varepsilon|\log \varepsilon|\right)} \mathrm{d} x \omega_{\varepsilon}(x, t) \geq 1-\frac{C_{1}}{\log |\log \varepsilon|} \quad \forall t \in[0, T] \quad \forall \varepsilon \in\left(0, \varepsilon_{1}\right] .
$$

This is the content of [5, Lemma 3.1] and it is an extension of the analogous result in [3], where the case without external field is considered. However, since the demonstration given in [5] is affected by an error, we provide here the correct proof, see "Appendix A."

The following proposition shows that in the limit $\varepsilon \rightarrow 0$ the center of vorticity performs a motion with constant speed along the $x_{1}=z$ axis.

Proposition 4.2. Under Assumption 2.1, for any $T>0$,

$$
\lim _{\varepsilon \rightarrow 0} \max _{t \in[0, T]}\left|B_{\varepsilon}(t)-\zeta(t)\right|=0,
$$

with $\zeta(t)$ as in Eq. (2.11).

Proof. Since $\Lambda_{\varepsilon}(t)$ is compact we can use Eq. (3.2) as in getting Eq. (3.17) and compute,

$$
\begin{aligned}
\dot{B}_{\varepsilon}(t)= & |\log \varepsilon| \frac{\mathrm{d}}{\mathrm{d} t} \int \mathrm{d} x x \omega_{\varepsilon}(x, t)=|\log \varepsilon| \int \mathrm{d} x \omega_{\varepsilon}(x, t)\left(u+F^{\varepsilon}\right)(x, t) \\
= & |\log \varepsilon| \int \mathrm{d} x \omega_{\varepsilon}(x, t) F^{\varepsilon}(x, t) \\
& +|\log \varepsilon| \int \mathrm{d} x \omega_{\varepsilon}(x, t) \int \mathrm{d} y[L(x, y)+\mathcal{R}(x, y)] \omega_{\varepsilon}(y, t),
\end{aligned}
$$

where we used Eq. (3.9) and $\int \mathrm{d} x \omega_{\varepsilon}(x, t) \widetilde{u}(x, t)=0$. In what follows, we fix $T>0$ and $k \in\left(0, \frac{1}{4}\right)$, and assume the parameter $\varepsilon$ so small in order that Eq. (4.1) does hold and that Eq. (3.8) implies $T_{\varepsilon}^{0}=T$. Therefore, from Eq. (4.3), (2.10), in view of Eqs. (3.5), (3.10), and (3.11), we have,

$$
\left|\dot{B}_{\varepsilon, 1}(t)-Q_{\varepsilon}(t)\right|+\left|\dot{B}_{\varepsilon, 2}(t)\right| \leq \frac{C}{|\log \varepsilon|} \quad \forall t \in[0, T]
$$

where

$$
Q_{\varepsilon}(t):=|\log \varepsilon| \int \mathrm{d} x \omega_{\varepsilon}(x, t) \frac{1}{4 \pi x_{2}} \int \mathrm{d} y \log \frac{1+|x-y|}{|x-y|} \omega_{\varepsilon}(y, t) .
$$

To determine the behavior of $Q_{\varepsilon}(t)$ as $\varepsilon \rightarrow 0$, we decompose,

$$
Q_{\varepsilon}(t)=Q_{\varepsilon}^{1}(t)+Q_{\varepsilon}^{2}(t)
$$

with

$$
Q_{\varepsilon}^{1}(t):=|\log \varepsilon| \int_{\Sigma\left(q_{\varepsilon}(t), \varepsilon|\log \varepsilon|\right)} \mathrm{d} x \omega_{\varepsilon}(x, t) \frac{1}{4 \pi x_{2}} \int_{\Sigma\left(q_{\varepsilon}(t), \varepsilon|\log \varepsilon|\right)} \mathrm{d} y \log \frac{1+|x-y|}{|x-y|} \omega_{\varepsilon}(y, t) .
$$


The rest $Q_{\varepsilon}^{2}(t)=Q_{\varepsilon}(t)-Q_{\varepsilon}^{1}(t)$ is the sum of three terms, each one is the integration of the same function, which in view of Eq. (3.11) is bounded by

$$
\mathcal{G}(x, y):=\frac{1}{2 \pi r_{0}} \log \frac{1+|x-y|}{|x-y|} \omega_{\varepsilon}(x, t) \omega_{\varepsilon}(y, t),
$$

and in the integration domain at least one between the $x$ and the $y$ variable is contained in the set $\Sigma\left(q_{\varepsilon}(t), \varepsilon|\log \varepsilon|\right)^{\complement}$. Therefore, since $\mathcal{G}$ is a symmetric function,

$$
Q_{\varepsilon}^{2}(t) \leq \frac{3|\log \varepsilon|}{2 \pi r_{0}} \int_{\Sigma\left(q_{\varepsilon}(t), \varepsilon|\log \varepsilon|\right)^{\mathrm{C}}} \mathrm{d} x \omega_{\varepsilon}(x, t) \int \mathrm{d} y \log \frac{1+|x-y|}{|x-y|} \omega_{\varepsilon}(y, t) \leq \frac{C}{\log |\log \varepsilon|},
$$

where we first bounded the dy-integral as done in Eq. (3.13), and then we used Eq. (4.1).

Concerning $Q_{\varepsilon}^{1}(t)$, we can obtain a lower bound for it, by inserting a lower bound to the function $\frac{1}{4 \pi x_{2}} \log \frac{1+|x-y|}{|x-y|}$ in the domain of integration and applying again Eq. (4.1),

$$
\begin{aligned}
Q_{\varepsilon}^{1}(t) & \geq \frac{|\log \varepsilon|}{4 \pi\left(q_{\varepsilon, 2}(t)+\varepsilon|\log \varepsilon|\right)} \log \frac{1+2 \varepsilon|\log \varepsilon|}{2 \varepsilon|\log \varepsilon|}\left(\int_{\Sigma\left(q_{\varepsilon}(t), \varepsilon|\log \varepsilon|\right)} \mathrm{d} x \omega_{\varepsilon}(x, t)\right)^{2} \\
& \geq \frac{|\log \varepsilon|}{4 \pi\left(q_{\varepsilon, 2}(t)+\varepsilon|\log \varepsilon|\right)} \log \frac{1+2 \varepsilon|\log \varepsilon|}{2 \varepsilon|\log \varepsilon|} \frac{1}{|\log \varepsilon|^{2}}\left(1-\frac{C_{1}}{\log |\log \varepsilon|}\right)^{2}
\end{aligned}
$$

On the other hand, by Eqs. (3.12) and (3.13), we can obtain an upper bound for $Q_{\varepsilon}^{1}(t)$,

$$
\begin{aligned}
Q_{\varepsilon}^{1}(t) & \leq \frac{1}{4 \pi\left(q_{\varepsilon, 2}(t)-\varepsilon|\log \varepsilon|\right)} \sup _{x} \int \mathrm{d} y \log \frac{1+|x-y|}{|x-y|} \omega_{\varepsilon}(y, t) \\
& \leq \frac{3 M}{2 \varepsilon^{2}|\log \varepsilon|\left(q_{\varepsilon, 2}(t)-\varepsilon|\log \varepsilon|\right)}\left\{\frac{\bar{\rho}^{2}}{2} \log \frac{1+\bar{\rho}}{\bar{\rho}}+\frac{1}{2} \int_{0}^{\bar{\rho}} \mathrm{d} \rho \frac{\rho}{1+\rho}\right\},
\end{aligned}
$$

with $\bar{\rho}$ such that $3 \pi \bar{\rho}^{2} M /\left(\varepsilon^{2}|\log \varepsilon|\right)=1 /|\log \varepsilon|$. Now, in view of Eq. (4.1), the disk $\Sigma\left(q_{\varepsilon}(t), \varepsilon|\log \varepsilon|\right)$ must have non-empty intersection with $\Lambda_{\varepsilon}(t)$. Therefore, since we are assuming $\varepsilon$ so small that $T_{\varepsilon}^{0}=T$, from Eq. (3.8) we deduce that

$$
\max _{t \in[0, T]}\left|q_{\varepsilon, 2}(t)-r_{0}\right| \leq \frac{C}{|\log \varepsilon|^{k}}+\varepsilon|\log \varepsilon| .
$$

We conclude that the right-hand side in both Eqs. (4.5) and (4.6) converges to $1 /\left(4 \pi r_{0}\right)$ as $\varepsilon \rightarrow 0$, so that, in view of Eq. (4.4),

$$
\lim _{\varepsilon \rightarrow 0} \max _{t \in[0, T]}\left|B_{\varepsilon, 1}(t)-\left(z_{0}+\frac{t}{4 \pi r_{0}}\right)\right|=0,
$$

which, together with Eq. (3.49), proves Eq. (4.2) (recall we fixed $a=1$ ).

From Eq. (4.1) and Proposition 4.2, the proof of item (2) of Theorem 2.2 is completed if we show that

$$
\lim _{\varepsilon \rightarrow 0} \sup _{t \in[0, T]}\left[B_{\varepsilon}(t)-q_{\varepsilon}(t)\right]=0
$$


(actually, by Eq. (4.7), the convergence of the second component is already known, but this does not shorten the proof). To this aim, we set $\Sigma_{t}=\Sigma\left(q_{\varepsilon}(t), \varepsilon|\log \varepsilon|\right)$ and compute,

$$
\begin{aligned}
\left|B_{\varepsilon}(t)-q_{\varepsilon}(t)\right| & \leq|\log \varepsilon| \int \mathrm{d} x\left|x-q_{\varepsilon}(t)\right| \omega_{\varepsilon}(x, t) \\
& =|\log \varepsilon| \int_{\Sigma_{t}} \mathrm{~d} x\left|x-q_{\varepsilon}(t)\right| \omega_{\varepsilon}(x, t)+|\log \varepsilon| \int_{\Sigma_{t}^{C}} \mathrm{~d} x\left|x-q_{\varepsilon}(t)\right| \omega_{\varepsilon}(x, t) \\
& \leq \varepsilon|\log \varepsilon|+\frac{C_{1}}{\log |\log \varepsilon|}\left|B_{\varepsilon}(t)-q_{\varepsilon}(t)\right|+|\log \varepsilon| \int_{\Sigma_{t}^{C}} \mathrm{~d} x\left|x-B_{\varepsilon}(t)\right| \omega_{\varepsilon}(x, t) .
\end{aligned}
$$

where we used Eq. (4.1). Therefore, by assuming $\varepsilon$ so small to have $2 C_{1} \leq \log |\log \varepsilon|$,

$$
\begin{aligned}
\left|B_{\varepsilon}(t)-q_{\varepsilon}(t)\right| & \leq 2 \varepsilon|\log \varepsilon|+2 \sqrt{|\log \varepsilon| \int_{\Sigma_{t}^{C}} \mathrm{~d} x \omega_{\varepsilon}(x, t)} \sqrt{|\log \varepsilon| \int_{\Sigma_{t}^{C}} \mathrm{~d} x\left|x-B_{\varepsilon}(t)\right|^{2} \omega_{\varepsilon}(x, t)} \\
& \leq 2 \varepsilon|\log \varepsilon|+2 \sqrt{\frac{C_{1}}{\log |\log \varepsilon|}} \sqrt{|\log \varepsilon| J_{\varepsilon}(t)},
\end{aligned}
$$

where we applied again Eq. (4.1), the Cauchy-Schwarz inequality, and introduced the moment of inertia with respect to center of vorticity defined as

$$
J_{\varepsilon}(t)=\int \mathrm{d} x\left|x-B_{\varepsilon}(t)\right|^{2} \omega_{\varepsilon}(x, t)
$$

Now, we claim that

$$
J_{\varepsilon}(t) \leq \frac{C}{|\log \varepsilon|} \quad \forall t \in[0, T]
$$

from which Eq. (4.9) follows in view of the above estimate on $\left|B_{\varepsilon}(t)-q_{\varepsilon}(t)\right|$. Compared with Lemma 3.2, the proof of Eq. (4.11) will require some more effort, due to the presence of the non-Lipschitz term Eq. (3.5). We compute the time derivative of $J_{\varepsilon}(t)$, by using Eq. (3.2),

$$
\dot{J}_{\varepsilon}(t)=2 \int \mathrm{d} x \omega_{\varepsilon}(x, t)\left(x-B_{\varepsilon}(t)\right) \cdot\left(u(x, t)+F^{\varepsilon}(x, t)-\dot{B}_{\varepsilon}(t)\right),
$$

so that, in view of Eq. (4.3),

$$
\begin{aligned}
\dot{J}_{\varepsilon}(t)= & 2 \int \mathrm{d} x \omega_{\varepsilon}(x, t)\left[u(x, t)-|\log \varepsilon| \int \mathrm{d} y \omega_{\varepsilon}(y, t) u(y, t)\right] \cdot\left(x-B_{\varepsilon}(t)\right) \\
& +2 \int \mathrm{d} x \omega_{\varepsilon}(x, t)\left[F^{\varepsilon}(x, t)-|\log \varepsilon| \int \mathrm{d} y \omega_{\varepsilon}(y, t) F^{\varepsilon}(y, t)\right] \cdot\left(x-B_{\varepsilon}(t)\right) .
\end{aligned}
$$

We consider first the term containing $F^{\varepsilon}$ and note that, by definition of $B_{\varepsilon}(t)$,

$$
\int \mathrm{d} x \omega_{\varepsilon}(x, t)\left(x-B_{\varepsilon}(t)\right) \cdot \int \mathrm{d} y \omega_{\varepsilon}(y, t) F^{\varepsilon}(y, t)=0, \quad \int \mathrm{d} x \omega_{\varepsilon}(x, t)\left(x-B_{\varepsilon}(t)\right) \cdot F^{\varepsilon}\left(B_{\varepsilon}(t), t\right)=0 .
$$

We thus obtain,

$$
\begin{aligned}
& 2\left|\int \mathrm{d} x \omega_{\varepsilon}(x, t)\left[F^{\varepsilon}(x, t)-|\log \varepsilon| \int \mathrm{d} y \omega_{\varepsilon}(y, t) F^{\varepsilon}(y, t)\right] \cdot\left(x-B_{\varepsilon}(t)\right)\right| \\
& \quad=2\left|\int \mathrm{d} x \omega_{\varepsilon}(x, t)\left[F^{\varepsilon}(x, t)-F^{\varepsilon}\left(B_{\varepsilon}(t), t\right)\right] \cdot\left(x-B_{\varepsilon}(t)\right)\right| \\
& \quad \leq 2 \int \mathrm{d} x \omega_{\varepsilon}(x, t) \frac{L}{|\log \varepsilon|}\left|x-B_{\varepsilon}(t)\right|^{2} \leq \frac{2 L}{|\log \varepsilon|} J_{\varepsilon}(t),
\end{aligned}
$$


where, in the last line, we used Eq. (2.10). For the term containing $u$, we have analogously,

$$
\int \mathrm{d} x \omega_{\varepsilon}(x, t)\left(x-B_{\varepsilon}(t)\right) \cdot \int \mathrm{d} y \omega_{\varepsilon}(y, t) u(y, t)=0 .
$$

Moreover, by the antisymmetry of $K$ and using Eq. (3.9),

$$
\int \mathrm{d} x \omega_{\varepsilon}(x, t) \widetilde{u}(x, t)=\int \mathrm{d} x \int \mathrm{d} y \omega_{\varepsilon}(x, t) \omega_{\varepsilon}(y, t) K(x-y)=0,
$$

so that, as $(x-y) \cdot K(x-y)=0$,

$$
\begin{aligned}
\int \mathrm{d} x \omega_{\varepsilon}(x, t) x \cdot \widetilde{u}(x, t) & =\int \mathrm{d} x \int \mathrm{d} y \omega_{\varepsilon}(x, t) \omega_{\varepsilon}(y, t) x \cdot K(x-y) \\
& =\int \mathrm{d} x \int \mathrm{d} y \omega_{\varepsilon}(x, t) \omega_{\varepsilon}(y, t) y \cdot K(x-y),
\end{aligned}
$$

which implies that also this integral is zero by the antisymmetry of $K$. Therefore,

$$
\begin{aligned}
& 2\left|\int \mathrm{d} x \omega_{\varepsilon}(x, t)\left[u(x, t)-|\log \varepsilon| \int \mathrm{d} y \omega_{\varepsilon}(y, t) u(y, t)\right] \cdot\left(x-B_{\varepsilon}(t)\right)\right| \\
& \quad \leq 2 \int \mathrm{d} x \omega_{\varepsilon}(x, t)\left|\int \mathrm{d} y L(x, y) \omega_{\varepsilon}(y, t)+\int \mathrm{d} y \mathcal{R}(x, y) \omega_{\varepsilon}(y, t)\right|\left|x-B_{\varepsilon}(t)\right| \\
& \quad \leq C \int \mathrm{d} x \omega_{\varepsilon}(x, t)\left|x-B_{\varepsilon}(t)\right| \leq \frac{C}{|\log \varepsilon|^{1 / 2}} \sqrt{J_{\varepsilon}(t)},
\end{aligned}
$$

where we have used Eq. (3.10) and Cauchy-Schwarz inequality.

In conclusion,

$$
\left|\dot{J}_{\varepsilon}(t)\right| \leq \frac{2 L}{|\log \varepsilon|} J_{\varepsilon}(t)+\frac{C}{|\log \varepsilon|^{1 / 2}} \sqrt{J_{\varepsilon}(t)}
$$

Recalling that the initial data imply $J_{\varepsilon}(0) \leq 4 \varepsilon^{2}$, this differential inequality implies Eq. (4.11). The proof of item (2) of Theorem 2.2 is thus completed.

Funding Open access funding provided by Università degli Studi di Roma La Sapienza within the CRUICARE Agreement.

Open Access. This article is licensed under a Creative Commons Attribution 4.0 International License, which permits use, sharing, adaptation, distribution and reproduction in any medium or format, as long as you give appropriate credit to the original author(s) and the source, provide a link to the Creative Commons licence, and indicate if changes were made. The images or other third party material in this article are included in the article's Creative Commons licence, unless indicated otherwise in a credit line to the material. If material is not included in the article's Creative Commons licence and your intended use is not permitted by statutory regulation or exceeds the permitted use, you will need to obtain permission directly from the copyright holder. To view a copy of this licence, visit http://creativecommons.org/licenses/by/4.0/.

Publisher's Note Springer Nature remains neutral with regard to jurisdictional claims in published maps and institutional affiliations.

\section{Appendix A: Proof of Lemma 4.1}

In the absence of external field, the proof of the concentration estimate Eq. (4.1) given in [3] is based on the conservation along the motion of the kinetic energy $E=\frac{1}{2} \int \mathrm{d} \boldsymbol{\xi}|\boldsymbol{u}(\boldsymbol{\xi}, t)|^{2}$, which in cylindrical coordinates $x=\left(x_{1}, x_{2}\right)=(z, r)$ takes the form

$$
E=\frac{1}{2} \int \mathrm{d} x 2 \pi x_{2}|u(x, t)|^{2} .
$$


More precisely, the assumptions on the initial vorticity, together with Eq. (1.7) and the conservation of the quantities

$$
M_{0}=\int \mathrm{d} x \omega_{\varepsilon}(x, t), \quad M_{2}=\int \mathrm{d} x x_{2}^{2} \omega_{\varepsilon}(x, t)
$$

allow to compute the asymptotic behavior as $\varepsilon \rightarrow 0$ of the energy $E$, from which the desired concentration estimate is deduced.

In the present case, the conservation law of $M_{0}$ is still valid, see Eq. (3.14). Concerning the variation of $M_{2}$, since $\omega_{\varepsilon}(x, t)$ has compact support, we can apply Eq. (3.2) with $f(x, t)=x_{2}^{2}$, so that

$$
\dot{M}_{2}=\int \mathrm{d} x \omega_{\varepsilon}(x, t) 2 x_{2} F_{2}^{\varepsilon}(x, t)
$$

which implies $\left|\dot{M}_{2}\right| \leq 2 C_{F}|\log \varepsilon|^{-3 / 2} \sqrt{M_{2}}$ in view of Assumption 2.1, item (b). Therefore, using also Eq. (2.9), $M_{2}(t) \leq M_{2}(0)+$ (const.) $|\log \varepsilon|^{-2} \leq\left(r_{0}+\varepsilon\right)^{2}|\log \varepsilon|^{-1}+$ (const.) $|\log \varepsilon|^{-2}$. Thus, $M_{2}$ is not conserved, but this only implies the presence of the extra term (const.) $|\log \varepsilon|^{-2}$ in the upper bound, which is easily seen to be irrelevant in the proof of $[3$, Thm. 1$]$.

Concerning the variation of $E$, we observe that in the proof of [3, Thm. 1] the energy conservation is used to deduce a lower bound of the form $E>C^{*} /|\log \varepsilon|+\left(\right.$ const.) $/|\log \varepsilon|^{2}$, because such estimate can be easily shown to be true for the energy computed at time zero. The crucial point in the argument is that $C^{*}$ is the same constant appearing in the leading term of an upper bound deduced for the energy $E$ at any time. Therefore, the proof given in [3] is valid also in the present case provided $|\dot{E}| \leq\left(\right.$ const.) $/|\log \varepsilon|^{2}$.

To compute $\dot{E}$, we introduce the stream function

$$
\Psi(x, t)=\int \mathrm{d} y S(x, y) \omega_{\varepsilon}(y, t),
$$

where the Green function $S(x, y)$ reads

$$
S(x, y):=\frac{x_{2} y_{2}}{2 \pi} \int_{0}^{\pi} \mathrm{d} \theta \frac{\cos \theta}{\sqrt{|x-y|^{2}+2 x_{2} y_{2}(1-\cos \theta)}},
$$

so that $u(x, t)=x_{2}^{-1} \nabla^{\perp} \Psi(x, t)$ and the energy takes the form (see, e.g., [3,11])

$$
E=\pi \int \mathrm{d} x \Psi(x, t) \omega_{\varepsilon}(x, t)=\pi \int \mathrm{d} x \int \mathrm{d} y S(x, y) \omega_{\varepsilon}(x, t) \omega_{\varepsilon}(y, t) .
$$

As before, since $\omega_{\varepsilon}(x, t)$ has compact support we can apply Eq. (3.2), so that, since $u \cdot \nabla \Psi=0$,

$$
\dot{E}=\pi \int \mathrm{d} x \omega_{\varepsilon}(x, t)\left(F^{\varepsilon} \cdot \nabla \Psi+\partial_{t} \Psi\right)(x, t),
$$

where, again from Eq. (3.2),

$$
\partial_{t} \Psi(x, t)=\int \mathrm{d} y \omega_{\varepsilon}(y, t)\left(u+F^{\varepsilon}\right)(y, t) \cdot \nabla_{y} S(x, y) .
$$

Since $S(x, y)$ is symmetric then $\Psi(y, t)=\int \mathrm{d} x S(x, y) \omega_{\varepsilon}(x, t)$, whence

$$
\begin{aligned}
\int \mathrm{d} x \omega_{\varepsilon}(x, t) \partial_{t} \Psi(x, t) & =\int \mathrm{d} x \omega_{\varepsilon}(x, t) \int \mathrm{d} y \omega_{\varepsilon}(y, t)\left(u+F^{\varepsilon}\right)(y, t) \cdot \nabla_{y} S(x, y) \\
& =\int \mathrm{d} y \omega_{\varepsilon}(y, t)\left(u+F^{\varepsilon}\right)(y, t) \cdot \nabla_{y} \int \mathrm{d} x S(x, y) \omega_{\varepsilon}(x, t) \\
& =\int \mathrm{d} y \omega_{\varepsilon}(y, t)\left(F^{\varepsilon} \cdot \nabla \Psi\right)(y, t),
\end{aligned}
$$


where we used again the orthogonality condition $u \cdot \nabla \Psi=0$. In conclusion,

$$
\dot{E}=2 \pi \int \mathrm{d} x \omega_{\varepsilon}(x, t)\left(F^{\varepsilon} \cdot \nabla \Psi\right)(x, t)=2 \pi \int \mathrm{d} x \int \mathrm{d} y \omega_{\varepsilon}(x, t) F^{\varepsilon}(x, t) \cdot \nabla_{x} S(x, y) \omega_{\varepsilon}(y, t) .
$$

Now, by direct computation [or simply recalling that $\nabla^{\perp} \Psi(x, t)=x_{2} u(x, t)$ together with Eqs. (2.2), $(2.5)$, and (2.6)],

$$
\begin{aligned}
& \frac{\partial S}{\partial x_{1}}=\frac{x_{2} y_{2}}{2 \pi} \int_{0}^{\pi} \mathrm{d} \theta \frac{\left(y_{1}-x_{1}\right) \cos \theta}{\left[|x-y|^{2}+2 x_{2} y_{2}(1-\cos \theta)\right]^{3 / 2}}, \\
& \frac{\partial S}{\partial x_{2}}=\frac{x_{2} y_{2}}{2 \pi} \int_{0}^{\pi} \mathrm{d} \theta \frac{y_{2}-x_{2} \cos \theta}{\left[|x-y|^{2}+2 x_{2} y_{2}(1-\cos \theta)\right]^{3 / 2}} .
\end{aligned}
$$

Therefore, letting

$$
a:=\frac{|x-y|}{\sqrt{x_{2} y_{2}}}
$$

and introducing the integrals

$$
I_{1}(a)=\int_{0}^{\pi} \mathrm{d} \theta \frac{\cos \theta}{\left[a^{2}+2(1-\cos \theta)\right]^{3 / 2}}, \quad I_{2}(a)=\int_{0}^{\pi} \mathrm{d} \theta \frac{1-\cos \theta}{\left[a^{2}+2(1-\cos \theta)\right]^{3 / 2}},
$$

the gradient $\nabla_{x} S$ reads

$$
\nabla_{x} S(x, y)=\frac{I_{1}(a)(y-x)}{2 \pi \sqrt{x_{2} y_{2}}}+\frac{I_{2}(a)}{2 \pi} \sqrt{\frac{y_{2}}{x_{2}}}\left(\begin{array}{l}
0 \\
1
\end{array}\right)=: A(x, y)+B(x, y)
$$

with $A(x, y)$ anti-symmetric, so that

$$
\begin{aligned}
\dot{E}= & \pi \int \mathrm{d} x \int \mathrm{d} y \omega_{\varepsilon}(x, t)\left[F^{\varepsilon}(x, t)-F^{\varepsilon}(y, t)\right] \cdot A(x, y) \omega_{\varepsilon}(y, t) \\
& +2 \pi \int \mathrm{d} x \int \mathrm{d} y \omega_{\varepsilon}(x, t) F^{\varepsilon}(x, t) \cdot B(x, y) \omega_{\varepsilon}(y, t) .
\end{aligned}
$$

In view of Assumption 2.1, item (b), and noticing that $\operatorname{div}\left(x_{2} F^{\varepsilon}\right)=0$ implies $F_{2}^{\varepsilon}\left(\left(x_{1}, 0\right), t\right)=0$, we deduce that

$$
\begin{aligned}
& \left|\left(F^{\varepsilon}(x, t)-F^{\varepsilon}(y, t)\right) \cdot A(x, y)\right| \leq \frac{L}{2 \pi|\log \varepsilon|} I_{1}(a) \frac{|y-x|^{2}}{\sqrt{x_{2} y_{2}}} \\
& \left|F^{\varepsilon}(x, t) \cdot B(x, y)\right|=\left|\left(F^{\varepsilon}(x, t)-F^{\varepsilon}\left(x_{1}, 0, t\right)\right) \cdot B(x, y)\right| \leq \frac{L}{2 \pi|\log \varepsilon|} I_{2}(a) \sqrt{x_{2} y_{2}} .
\end{aligned}
$$

We now recall the following estimates, see, e.g., [18, Appendix],

$$
\begin{aligned}
I_{1}(a) & \leq \int_{0}^{\pi} \mathrm{d} \theta \frac{\cos \frac{\theta}{2}}{\left[a^{2}+2(1-\cos \theta)\right]^{3 / 2}}=\int_{0}^{\pi} \mathrm{d} \theta \frac{\cos \frac{\theta}{2}}{\left[a^{2}+4 \sin ^{2} \frac{\theta}{2}\right]^{3 / 2}}=\frac{2}{a^{2} \sqrt{a^{2}+4}}, \\
I_{2}(a) & \leq \frac{1}{2} \log \left(2+\sqrt{a^{2}+4}\right)-\frac{1}{2} \log a+(\text { const. }) \\
& \left.=\frac{1}{2} \log \left(2 \sqrt{x_{2} y_{2}}+\sqrt{b+4 x_{2} y_{2}}\right)-\frac{1}{4} \log b+\text { (const. }\right),
\end{aligned}
$$

where in the last identity we introduced the quantity $b=|x-y|^{2}$ as in [3]. Therefore,

$$
\left|\left(F^{\varepsilon}(x, t)-F^{\varepsilon}(y, t)\right) \cdot A(x, y)\right| \leq \frac{\text { (const. })}{|\log \varepsilon|} \sqrt{x_{2} y_{2}},
$$




$$
\left|F^{\varepsilon}(x, t) \cdot B(x, y)\right| \leq \frac{(\text { const. })}{|\log \varepsilon|} \sqrt{x_{2} y_{2}}\left[\log \left(2 \sqrt{x_{2} y_{2}}+\sqrt{b+4 x_{2} y_{2}}\right)-\frac{1}{2} \log b+(\text { const. })\right],
$$

so that

$$
|\dot{E}| \leq \frac{(\text { const. })}{|\log \varepsilon|} \int \mathrm{d} x \int \mathrm{d} y \omega_{\varepsilon}(x, t) \omega_{\varepsilon}(y, t) \sqrt{x_{2} y_{2}}\left\{(\text { const. })+\log \left(2 \sqrt{x_{2} y_{2}}+\sqrt{b+4 x_{2} y_{2}}\right)-\frac{1}{2} \log b\right\} .
$$

Except for the prefactor (const.) $/|\log \varepsilon|$, the right-hand side in the above inequality equals the first upper bound on $E$ appearing in [3, Eq. (2.30)], from which the estimate $E \leq$ (const.) $/|\log \varepsilon|$ is deduced. We conclude that $|\dot{E}| \leq$ (const.) $/|\log \varepsilon|^{2}$ as required.

\section{Appendix B: Proof of Lemma 2.3}

Recalling Eq. (A.1), we have

$$
\begin{aligned}
G^{i, \varepsilon}(x, t) & =\int \mathrm{d} y H(x, y) \bar{\omega}_{i, \varepsilon}(y, t) \\
& =\frac{1}{x_{2}} \int \mathrm{d} y \nabla_{x}^{\perp} S(x, y) \bar{\omega}_{i, \varepsilon}(y, t),
\end{aligned}
$$

where

$$
\bar{\omega}_{i, \varepsilon}(y, t):=\sum_{j \neq i} \omega_{j, \varepsilon}(y, t),
$$

with, in view of mass conservation and Eq. (1.15),

$$
\int \mathrm{d} y\left|\bar{\omega}_{i, \varepsilon}(y, t)\right| \leq \frac{A}{|\log \varepsilon|}, \quad A:=\sum_{j}\left|a_{j}\right| .
$$

Recalling the definition Eq. (2.12) of $T_{\varepsilon}$, we let

$$
\begin{aligned}
& \Omega_{i}:=\left\{x:\left|x_{2}-r_{i}\right| \leq R\right\}, \\
& \tilde{\Omega}_{i}:=\bigcup_{j \neq i} \Omega_{j},
\end{aligned}
$$

so that

$$
\Lambda_{i, \varepsilon}(t) \subset \Omega_{i}, \quad \tilde{\Lambda}_{i, \varepsilon}(t):=\operatorname{supp} \bar{\omega}_{i, \varepsilon}(\cdot, t) \subset \tilde{\Omega}_{i} \quad \forall t \in\left[0, T_{\varepsilon}\right]
$$

and

$$
\operatorname{dist}\left(\Omega_{i}, \tilde{\Omega}_{i}\right) \geq 2(D-R), \quad \tilde{\Omega}_{i} \subset\left\{x: D \leq x_{2} \leq \max _{j} r_{j}+R\right\},
$$

with $D$ as in Eq. (1.12). In particular, from the explicit expression of $H(x, y)=x_{2}^{-1} \nabla_{x}^{\perp} S(x, y)$, see Eqs. (2.5) and (2.6), or (A.2), it is easily seen that (below $D_{x} H(x, y)$ denotes the Jacobian matrix of $H(x, y)$ with respect to the variable $x)$

$$
|H(x, y)|+\left\|D_{x} H(x, y)\right\| \leq C \quad \forall(x, y) \in \Omega_{i} \times \tilde{\Omega}_{i},
$$

whence, in view of Eq. (B.2),

$$
\begin{aligned}
\left|G^{i, \varepsilon}(x, t)\right| & \leq \frac{C}{|\log \varepsilon|}, \\
\left|G^{i, \varepsilon}(x, t)-G^{i, \varepsilon}(y, t)\right| & \leq \frac{C}{|\log \varepsilon|}|x-y| \quad \forall x, y \in \Omega_{i} \quad \forall t \in\left[0, T_{\varepsilon}\right] .
\end{aligned}
$$


Therefore, the auxiliary field $F^{i, \varepsilon}(x, t)$ as claimed in the lemma can be obtained by setting

$$
F^{i, \varepsilon}(x, t)=\frac{1}{x_{2}} \int \mathrm{d} y \nabla_{x}^{\perp} \tilde{S}(x, y) \bar{\omega}_{i, \varepsilon}(y, t),
$$

where $\tilde{S}(x, y)$ is any mollification of $S(x, y)$ which is bounded, globally Lipschitz, and such that $\tilde{S}(x, y)=$ $S(x, y)$ whenever $(x, y) \in \Omega_{i} \times \tilde{\Omega}_{i}$.

\section{References}

[1] Ambrosetti, A., Struwe, M.: Existence of steady rings in an ideal fluid. Arch. Ration. Mech. Anal. 108, 97-108 (1989)

[2] Bedrossian, J., Germain, P., Harrop-Griffiths, B.: Vortex filament solutions of the Navier-Stokes equations. Preprint arXiv:1809.04109

[3] Benedetto, D., Caglioti, E., Marchioro, C.: On the motion of a vortex ring with a sharply concentrate vorticity. Math. Methods Appl. Sci. 23, 147-168 (2000)

[4] Buttà, P., Marchioro, C.: Long time evolution of concentrated Euler flows with planar symmetry. SIAM J. Math. Anal. 50, 735-760 (2018)

[5] Buttà, P., Marchioro, C.: Time evolution of concentrated vortex rings. J. Math. Fluid Mech. 22, Article number 19 (2020)

[6] Cavallaro, G., Marchioro, C.: Time evolution of vortex rings with large radius and very concentrated vorticity. J. Math. Phys. 62, 053102, 20 pp. (2021)

[7] Cetrone, D., Serafini, G.: Long time evolution of fluids with concentrated vorticity and convergence to the point-vortex model. Rendiconti di Matematica e delle sue applicazioni 39, 29-78 (2018)

[8] Colagrossi, A., Graziani, G., Pulvirenti, M.: Particles for fluids: SPH versus vortex methods. Math. Mech. Complex Syst. 2, 45-70 (2014)

[9] Fraenkel, L.E.: On steady vortex rings of small cross-section in an ideal fluid. Proc. Roy. Soc. Lond. A. 316, 29-62 (1970)

[10] Fraenkel, L.E., Berger, M.S.: A global theory of steady vortex rings in an ideal fluid. Acta Math. 132, 13-51 (1974)

[11] Friedman, A.: Variational Principles and Free-Boundary Problems. Wiley, New York (1982)

[12] Gallay, T.: Interaction of vortices in weakly viscous planar flows. Arch. Ration. Mech. Anal. 200, 445-490 (2011)

[13] Gallay, T., Šverák, V.: Remarks on the Cauchy problem for the axisymmetric Navier-Stokes equations. Confluentes Math. 7, 67-92 (2015)

[14] Gallay, T., Šverák, V.: Uniqueness of axisymmetric viscous flows originating from circular vortex filaments. Ann. Sci. Éc. Norm. Supér. 52, 1025-1071 (2019)

[15] Majda, A., Bertozzi, A.: Vorticity and Incompressible Flow. Cambridge Texts in Applied Mathematics, Cambridge University Press, Cambridge (2002)

[16] Marchioro, C.: On the vanishing viscosity limit for two-dimensional Navier-Stokes equations with singular initial data. Math. Methods Appl. Sci. 12, 463-470 (1990)

[17] Marchioro, C.: On the inviscid limit for a fluid with a concentrated vorticity. Commun. Math. Phys. 196, 53-65 (1998)

[18] Marchioro, C.: Large smoke rings with concentrated vorticity. J. Math. Phys. 40, 869-883 (1999)

[19] Marchioro, C., Negrini, P.: On a dynamical system related to fluid mechanics. NoDEA Nonlinear Differ. Equ. Appl. 6, 473-499 (1999)

[20] Marchioro, C.: Vanishing viscosity limit for an incompressible fluid with concentrated vorticity. J. Math. Phys. 48, 065302, 16 pp. (2007)

[21] Marchioro, C., Pulvirenti, M.: Mathematical Theory of Incompressible Non-viscous Fluids. Applied Mathematical Sciences, vol. 96. Springer, New York (1994)

[22] Shariff, K., Leonard, A.: Vortex rings. Annu. Rev. Fluid Mech. 24, 235-279 (1972)

Paolo Buttà, Guido Cavallaro and Carlo Marchioro

Dipartimento di Matematica

Sapienza Università di Roma

P.le Aldo Moro 5

00185 Roma

Italy

e-mail: cavallar@mat.uniroma1.it

Paolo Buttà

e-mail: butta@mat.uniroma1.it 
Carlo Marchioro

e-mail: marchior@mat.uniroma1.it

Carlo Marchioro

International Research Center M\&MOCS

Università di L'Aquila

Palazzo Caetani

04012 Cisterna di Latina LT

Italy

(Received: February 16, 2021; revised: February 2, 2022; accepted: February 5, 2022) 